Check for updates

Cite this: RSC Adv., 2019, 9, 37788

Received 23rd September 2019 Accepted 11th November 2019

DOI: 10.1039/c9ra07712

rsc.li/rsc-advances

\section{Allylation of isatin-derived N-Boc-hydrazones followed by Pd-catalyzed carboamination reaction: an entry to 3-spiro-pyrazolidyl-oxindoles $\uparrow$}

\author{
Stefano Gazzotti, (D) Marco Manenti, Leonardo Lo Presti (iD) and Alessandra Silvani \\ The indium-mediated allylation of novel 3-(2-Boc-hydrazono)indolin-2-one derivatives, followed by \\ a palladium-catalysed carboamination reaction, is described to afford unprecedented spirocyclic \\ oxindoles in good yields. The method provides an efficient access to both cis and trans diastereoisomers \\ of highly functionalized compounds, bearing an N-Boc, 5-substituted pyrazolidine ring at the C3- \\ oxindole spiro junction. The versatility of the method is fully demonstrated starting from a series of \\ substituted isatins and employing a variety of aryl halides in the key cyclization step.
}

\section{Introduction}

The pyrazolidine five-membered ring, characterized by the presence of two adjacent nitrogen atoms, can be considered as a cyclic hydrazine moiety. Among natural products containing a nitrogen-nitrogen bond, ${ }^{1}$ such a heterocycle is displayed by the highly unusual alkaloid garceine $(\mathbf{I}),{ }^{2}$ isolated from Lotus garcinii in 2001, but not investigated for biological properties because of insufficient material (Fig. 1).

Owing to their particular stability and, at the same time, peculiar electronic properties, pyrazolidines and their derivatives are potentially capable of providing improved physicochemical properties in their interaction with biological systems. ${ }^{3}$ Indeed, this heterocyclic ring is present as a partial structure in a number of synthetic compounds, displaying a wide range of bioactivities, such as, for example, antibacterial (II), ${ }^{4}$ anticancer (III) ${ }^{5}$ and anticonvulsant (IV). ${ }^{6}$

In addition, pyrazolidine-amino acid derivatives, acting as azaproline analogues, have been shown to have application as peptidomimetics, displaying inhibitory activities against enzymes (serine peptidase dipeptidyl IV inhibitor, $\mathbf{V})^{7}$ and receptors (VLA-4 antagonist, VI). ${ }^{8}$

Despite a recurrent interest in pyrazolidine 3,5-diones ${ }^{9-11}$ and N,N-disubstituted derivatives, ${ }^{12}$ other kinds of pyrazolidinebased frameworks have received minor attention, and their application in the context of drug discovery has yet to be fully explored. Over the last decade the synthetic effort towards

Dipartimento di Chimica, Universitá di Milano, Via Golgi 19, Milano, 20133, Italy. E-mail: alessandra.silvani@unimi.it

$\dagger$ Electronic supplementary information (ESI) available: ${ }^{1} \mathrm{H}$ and ${ }^{13} \mathrm{C}$ NMR spectra for all new compounds; ${ }^{1} \mathrm{H}^{-}{ }^{1} \mathrm{H}$ ROESY NMR for compounds $6 \mathbf{a}$ and $6 \mathbf{a}^{\prime}$; full discussion of the crystallographic results, including information on crystal packing, for compound 6f' (PDF). CCDC 1938731. For ESI and crystallographic data in CIF or other electronic format see DOI: 10.1039/c9ra07712j highly functionalized pyrazolidine derivatives has greatly increased, leading to the development of efficient protocols, such as cycloadditions of hydrazones and olefins, ${ }^{13}$ 1,3-dipolar cycloadditions of azomethine imines, ${ }^{\mathbf{1 4}}$ carboamination reactions, ${ }^{15}$ and amination reactions of allenes. ${ }^{\mathbf{1 6}}$

As part of our interest in the synthesis of 3,3-disubstituted oxindole derivatives and related spirocompounds, ${ }^{17}$ we looked into the biological significance of the pyrazolidine ring, conceiving its combination with the relevant oxindole nucleus, by means of a spiro arrangement of the two ring systems. The conjugation of privileged heterocycles into spiro structures is a challenging application of the molecular hybridization concept, ${ }^{18}$ a viable and effective approach envisioning the rational design of new functional compounds through the structural fusion of two pharmacophoric subunits into one chemical entity. Since spiro compounds have an intrinsic threedimensionality, they are able to access unexplored chemical space, often displaying improved biological interactions and being more likely to be successfully developed as drugs. ${ }^{19}$

At the best of our knowledge, only a recent work describes spirocyclic pyrazolidines, achieved by means of a gold-catalyzed
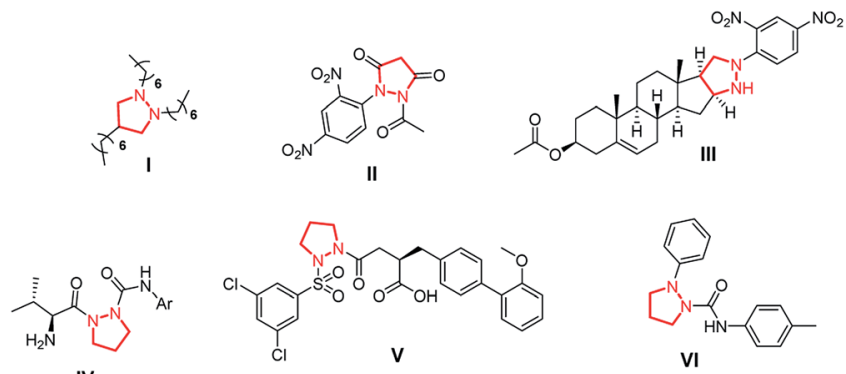

IV

Fig. 1 Examples of natural products and bioactive agents containing the pyrazolidine ring. 
three-component spirocyclization, starting from alkynols, hydrazines and carbonyl components, mainly aldehydes. ${ }^{\mathbf{2 0}}$

No methods have been reported for the preparation of pyrazolidines derived from cyclic ketones and, of course, of oxindoles bearing an $\mathrm{N}$-jointed pyrazolidine ring at the key $\mathrm{C} 3$ position.

Relying on our previous experience with isatin-derived ketimines and 3-amino substituted oxindoles, ${ }^{17}$ we envisioned 3-allyl-3hydrazinylindolin-2-ones 5, obtainable from 3-hydrazonoindolin-2ones 4, as suitable substrates for a palladium-catalysed carboamination reaction, aimed to the synthesis of the unprecedented $1^{\prime}, 5^{\prime}$-disubstituted spiro[indoline-3,3'-pyrazolidin]-2-one scaffold 6 (Scheme 1).

Herein, we report the synthesis of a large family of highly functionalized $1^{\prime}$-Boc, $5^{\prime}$-arylmethyl spiro[indoline-3,3'-pyrazolidin] -2-ones, attainable as separable $3^{\prime}-5^{\prime}$-cis and $3^{\prime}-5^{\prime}$-trans diastereoisomers, thus demonstrating for the first time the suitability of the Pd-catalysed carboamination reaction of hydrazine derivatives for the synthesis of spiro compounds.

\section{Results and discussion}

We started our investigation by taking into consideration the already known isatin-derived $N$-tosyl hydrazone $\mathbf{1}$ and the corresponding imino allylation product $2,{ }^{21}$ besides 4 -bromo-1, $1^{\prime}$ biphenyl as aryl halide. We tested various reaction conditions for the Pd-catalysed carboamination step. However, treatment of compound 2 with different $\mathrm{Pd}$ catalysts $\left(\mathrm{Pd}(\mathrm{OAc})_{2}, \mathrm{Pd}_{2}(\mathrm{dba})_{3}\right)$ in presence of various ligands (dppe, dpe-phos, BINAP) afforded high amounts of compounds $\mathbf{3} \mathbf{a}$ and $\mathbf{3} \mathbf{b}$, instead of the expected spiro compound (Scheme 2).

Likely, $N$-tosyl hydrazine 2 is not stable in the basic reaction conditions employed and, due to the presence of $\mathrm{NaO}^{t} \mathrm{Bu}$, it easily decomposes, ejecting the tosyl anion and forming a diazonium anion. Loss of molecular nitrogen results in

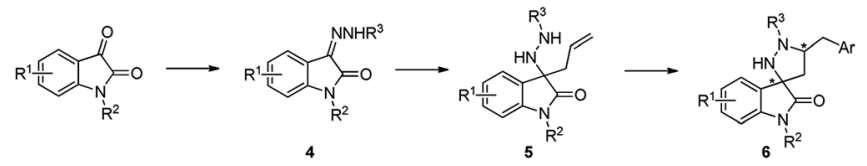

Scheme 1 Aim of the work.

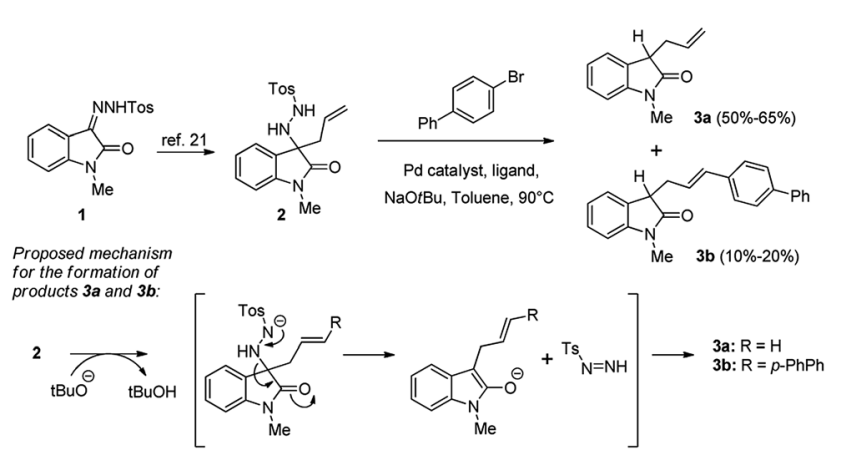

Scheme 2 Reaction studies on $N$-tosyl hydrazone 1 protonation of the substrate, affording the already known compound 3a. ${ }^{22}$ The formation of moderate amounts of compound $\mathbf{3 b}$ can be explained as the result of a standard Heck reaction between $3 \mathbf{a}$ and the 4-bromo-1,1'-biphenyl.

Thinking about more appropriate hydrazone N-substituents, we turned our attention to reaction of $N$-benzyl isatin with tertbutyl carbazate. After refluxing for two hours in ethanol, the unprecedented N-Boc hydrazone 4a was obtained in nearly quantitative yield (Table 1 , reaction). For the subsequent allylation reaction of $\mathbf{4 a}$, we initially explored the cheap tin powder, in combination with allyl bromide in a simple Barbier-type onepot procedure, which avoids the use of the toxic allylic tributyltin reagent. ${ }^{23}$ The reaction proved to be unsuccessful in $\mathrm{MeOH}$ (Table 1, entry 1), while it afforded the desired N-Boc allyl hydrazine derivative $5 \mathbf{a}$ when the solvent was changed to THF (entry 2).

Aiming at improving the yield and, at the same time, at experimenting an environmentally benign chemical process, we then looked at the indium-promoted allylation in aqueous media. ${ }^{21}$ Unlike most Barbier-type allylation methods, indiummediated reactions are insensitive to moisture, relying on facile and practical reaction conditions coupled with minimal side reactions. Treatment of a $\mathrm{THF} / \mathrm{NH}_{4} \mathrm{Cl}$ (aq. saturated) solution of hydrazone $4 \mathbf{a}$ with allyl bromide in the presence of indium afforded compound 5a only in traces (entry 3 ). The yield improved when the reaction was conducted in a $3 / 1 \mathrm{MeOH} /$ $\mathrm{NH}_{4} \mathrm{Cl}$ (aq. saturated) solution (entry 4) and it was ultimately satisfying when only $\mathrm{MeOH}$ was adopted as a solvent (entry 5). An excess of allyl bromide and indium powder proved to be necessary for an almost quantitative yield, probably due to progressive metal inactivation.

Once a multi-gram scale procedure for the preparation of the hydrazine substrate 5a was achieved, we moved our attention on the key Pd-catalysed carboamination reaction. Among all possible variables that can be considered for the screening of

Table 1 Synthesis of $\mathrm{N}$-Boc hydrazine derivative $5 \mathrm{a}^{a}$

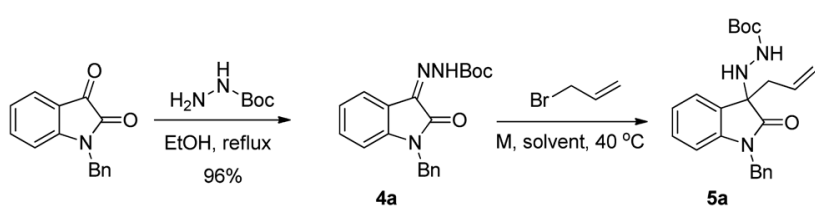

\begin{tabular}{lllll}
\hline Entry & M (powder) & Solvent & $T(\mathrm{~h})$ & Yield $^{b}(\%)$ \\
\hline 1 & $\mathrm{Sn}$ & $\mathrm{MeOH}$ & 48 & $<5$ \\
2 & $\mathrm{Sn}$ & $\mathrm{THF}$ & 48 & 70 \\
3 & In & $\mathrm{THF} / \mathrm{NH}_{4} \mathrm{Cl}$ sat $1: 1$ & 6 & $<5$ \\
4 & In & $\mathrm{MeOH} / \mathrm{NH}_{4} \mathrm{Cl}$ sat $3: 1$ & 72 & 40 \\
5 & In & $\mathrm{MeOH}$ & 3 & 94
\end{tabular}

${ }^{a}$ Compound 4a was prepared starting from $N$-benzyl isatin $(2 \mathrm{mmol})$, tert-butyl carbazate $(2 \mathrm{mmol})$, in ethanol $(0.3 \mathrm{M})$, at reflux for two hours. All allylation reactions were conducted with 4 a $(0.25 \mathrm{mmol})$, allyl bromide $(0.5 \mathrm{mmol})$ and metal powder $(0.5 \mathrm{mmol}) .{ }^{b}$ Isolated yields. 
the reaction conditions, we chose to focus preliminary on $\mathrm{Pd}$ complexes and ligand additives. We investigated the effect of $\operatorname{Pd}(0)$ - and $\operatorname{Pd}(\mathrm{II})$-based complexes and of several phosphines, at various degrees of basicity and steric encumbrance. After some preliminary screening on solvent and temperature, all reactions were carried out in toluene, at $90{ }^{\circ} \mathrm{C}$, using $\mathrm{NaO}^{t} \mathrm{Bu}$ as the base and 4-bromo-1,1'-biphenyl as reference aryl halide (Table 2).

We started our investigation from $\mathrm{Pd}(\mathrm{OAc})_{2}$ catalyst, coupled with various phosphine-based ligands. Reaction employing dpephos failed to give the desired spiro products, affording only compound 7 from the competing Heck arylation, and compound 8, from the Pd-promoted isomerization of the substrate 5a double bond (entry 1). Switching to triscyclohexylphospine (TCP), we were pleased to observe the formation of diastereoisomeric $\mathbf{6 a}$ and $\mathbf{6 a}^{\prime}$ target compounds (dr 1:2), together with a considerable amount of byproduct $\mathbf{9 ,}{ }^{\mathbf{2 4}}$ deriving from decomposition of the starting N-Boc-hydrazine (entry 2). A significant yield improvement and reduction of reaction times was achieved exploiting tris(o-tolyl)phosphine (TTP) or tris(2furyl)phosphine (TFP), but without any increase of diastereoisomeric ratios (entries 3 and 4). Switching to $\mathrm{PdCl}_{2}(\mathrm{MeCN})_{2}$, $\mathrm{PdCl}_{2}\left(\mathrm{PPh}_{3}\right)_{2}$ or $\mathrm{Pd}\left(\mathrm{PPh}_{3}\right)_{4}$ complexes did not lead to any contribution, in terms of better yield or dr (entries 5-9). On the other hand, $\mathrm{Pd}_{2}(\mathrm{dba})_{3}$ proved to be the catalyst of choice, both when paired with TTP or with X-phos, affording the desired 3spiro-pyrazolidyl-oxindoles $\mathbf{6 a}$ and $\mathbf{6 a}^{\prime}$ in excellent yield, albeit in negligible $\mathrm{dr}$ (entries 10-11). The known propensity of the
$\mathrm{Pd}_{2}(\mathrm{dba})_{3}$ complex to reduce the rate of $\beta$-hydrogen elimination in "PdII- $\sigma$-alkyl" complexes, ${ }^{25}$ can likely explain the ability of this catalyst to drive the reaction towards the intramolecular key carboamination, rather than towards the competing standard intermolecular Heck reaction.

Diastereoisomeric 3-spiro-pyrazolidyl-oxindoles $\mathbf{6 a}$ and $\mathbf{6} \mathbf{a}^{\prime}$ proved to be easily separable by flash chromatography, allowing a full mono- and bidimensional NMR characterization, including determination of the relative stereochemistry. In particular, NOESY experiments allowed assigning the $3^{\prime}-5^{\prime}$-trans configuration to diastereoisomer $\mathbf{6 a}$ and the $3^{\prime}-5^{\prime}$-cis configuration to diastereoisomer $\mathbf{6} \mathbf{a}^{\prime}$. Diagnostic NOE interactions between oxindole $\mathrm{H}-4$ and selected pyrazolidine protons were identified for both diastereoisomers (Fig. 2).

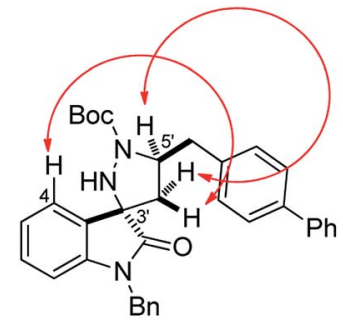

(3'-5'-trans)-6a

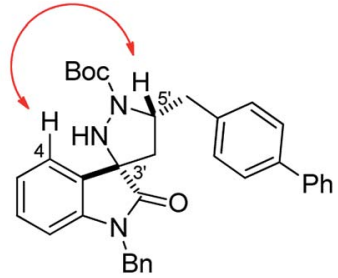

$\left(3^{\prime}-5 '-c i s\right)-6 a^{\prime}$
Fig. 2 Diagnostic NOE contacts for compounds $6 a$ and $6 a^{\prime}$.

Table 2 Screening of the $\mathrm{Pd}$-catalysed carboamination reaction conditions on hydrazine derivative $5 \mathrm{a}^{a}$

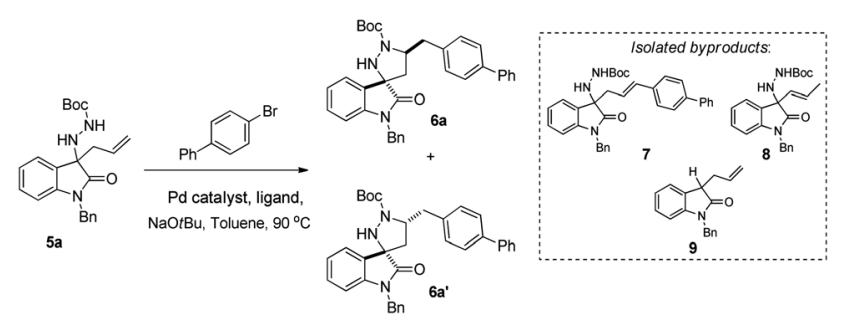

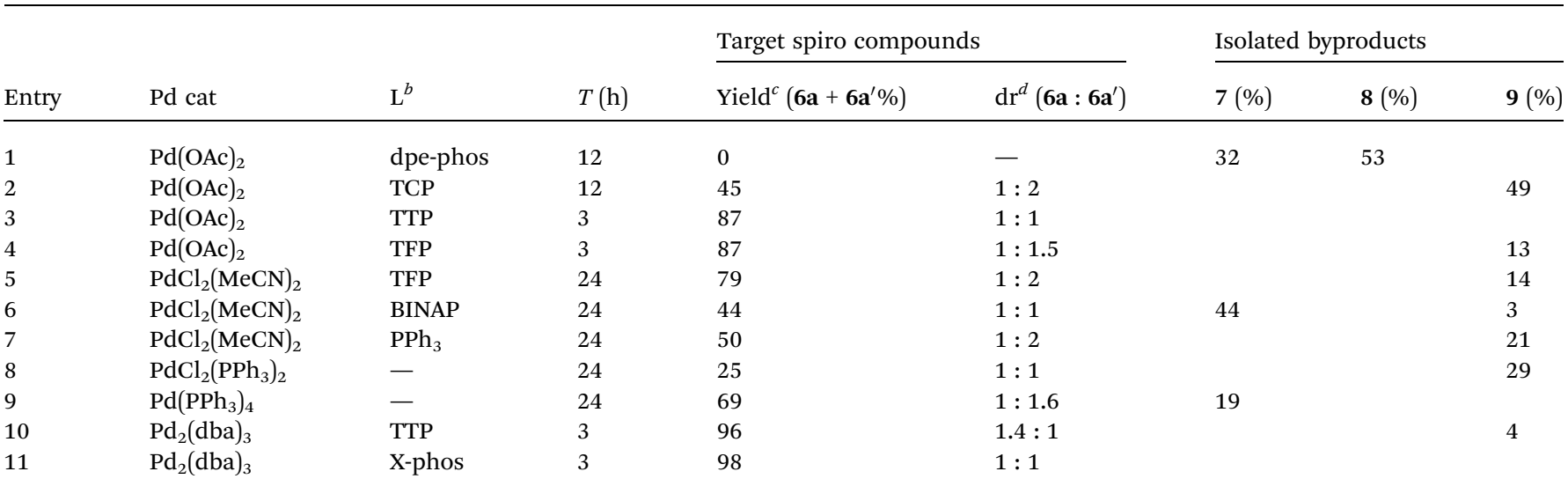

${ }^{a}$ Reactions were carried out on a $0.25 \mathrm{mmol}$ scale, with 1.0 equiv. of $5 \mathrm{a}, 1.3$ equiv. of 4 -bromo-1, $1^{\prime}$-biphenyl, $1.3 \mathrm{equiv}$. of $\mathrm{NaO}{ }^{t} \mathrm{Bu}, 4 \mathrm{~mol} \%$ of $\mathrm{Pd}$ catalyst, $4 \mathrm{~mol} \%$ of ligand, toluene $(0.20 \mathrm{M}) .{ }^{b}$ Dpe-phos = bis $\left[\left(2\right.\right.$-diphenylphosphino)phenyl]ether; BINAP= $( \pm)-2,2^{\prime}-$ bis $($ diphenylphosphino $)-1,1^{\prime}-$ binaphthalene; TFP $=\operatorname{tris}(2$-furyl)phosphine; TCP $=$ triscyclohexylphospine; TTP $=$ tris $(o$-tolyl)phosphine; X-phos $=2$-dicyclohexylphosphino$2^{\prime}, 4^{\prime}, 6^{\prime}$-triisopropylbiphenyl. ${ }^{c}$ Sum of the isolated yields for each diastereoisomer. ${ }^{d}$ Determined by ${ }^{1} \mathrm{H}$ NMR of the crude reaction product. 
In order to evaluate possible improvements in $\mathrm{dr}$, we made some focused modifications to the N-Boc hydrazine substrate. Aiming at exploring the effects of more steric constraints in the transition states, we selected $\mathrm{N}, \mathrm{N}^{2}$-Boc hydrazine 10 and 2,2dimethylbut-3-en-1-yl hydrazine 12, easily prepared from compounds $\mathbf{5 a}$ and $\mathbf{4 a}$, respectively (Scheme 3 ).

From the Pd-catalysed reaction on substrate 10, the monoBoc spiro derivatives $\mathbf{6 a}$ and $\mathbf{6} \mathbf{a}^{\prime}$ could be recovered in the usual dr, together with a substantial amount of the Heck
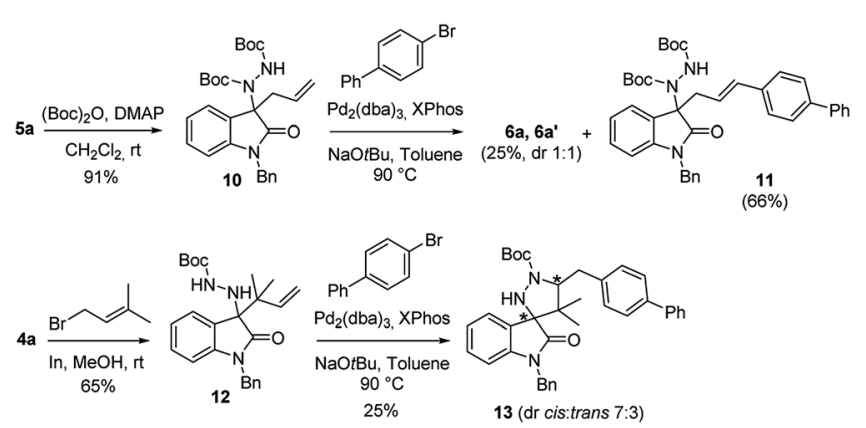

Scheme 3 Pd-catalysed carboamination reaction on hydrazine derivatives 10 and 12 .

Table 3 Generality of the substrate scope ${ }^{a}$
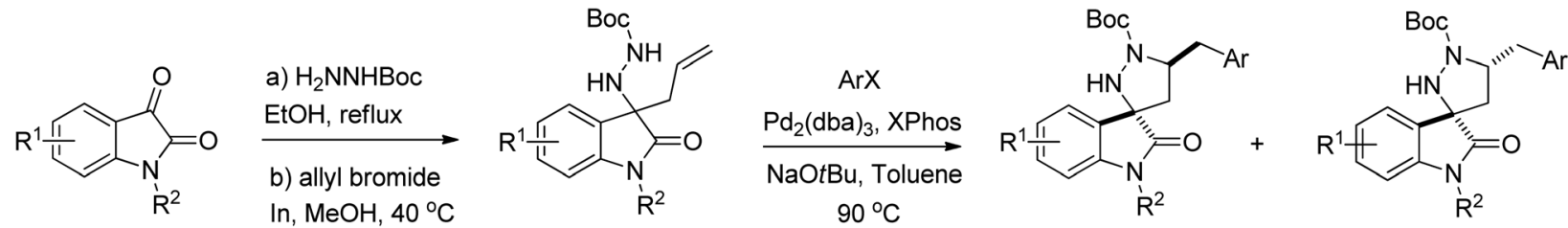

\begin{tabular}{|c|c|c|c|c|c|c|c|}
\hline Entry & $5\left(\mathrm{R}^{1}, \mathrm{R}^{2}\right)$ & Yield $(5 \%)^{b}$ & ArX & $T(\mathrm{~h})$ & 6 & Yield $(6 \%)^{c}$ & $\operatorname{dr}\left(\mathbf{6}: 6^{\prime}\right)^{d}$ \\
\hline 1 & $\mathbf{5 a}(\mathrm{H}, \mathrm{Bn})$ & 94 & $p$-Br-biphenyl & 3 & $6 a, 6 \mathbf{a}^{\prime}$ & 98 & $1: 1$ \\
\hline 2 & $\mathbf{5 a}(\mathrm{H}, \mathrm{Bn})$ & 94 & $p$-Br-anisole & 6 & $6 \mathbf{b}, 6 \mathbf{b}^{\prime}$ & 98 & $1.4: 1$ \\
\hline 4 & $\mathbf{5 a}(\mathrm{H}, \mathrm{Bn})$ & 94 & $p$-Br-benzophenone & 4 & $6 d, 6 d^{\prime}$ & 92 & $1: 1$ \\
\hline 5 & $5 \mathbf{a}(\mathrm{H}, \mathrm{Bn})$ & 94 & $p$-Br-acetophenone & 3 & $6 e, 6 e^{\prime}$ & 98 & $1: 1$ \\
\hline 6 & $5 a(H, B n)$ & 94 & $o$-Br-toluene & 10 & $6 f, 6 f^{\prime}$ & 98 & $1: 1.6$ \\
\hline 9 & $\mathbf{5 b}(\mathrm{H}, \mathrm{Me})$ & 82 & $p$-Br-biphenyl & 12 & $6 \mathbf{i}, \mathbf{6 \mathbf { i } ^ { \prime }}$ & 53 & $1.5: 1$ \\
\hline 10 & $5 \mathbf{c}\left(\mathrm{H}, 3,4\right.$-diCl- $\left.\mathrm{Ph}(\mathrm{CH})_{2} \mathrm{CH}_{2}\right)$ & 93 & $p$-Br-biphenyl & 12 & $6 \mathbf{j}, 6 \mathbf{j}^{\prime}$ & 69 & $1: 1$ \\
\hline 11 & $\mathbf{5 d}(5-\mathrm{Me}, \mathrm{Bn})$ & 98 & $p$-Br-biphenyl & 12 & $6 \mathbf{k}, \mathbf{6} \mathbf{k}^{\prime}$ & 50 & $1.4: 1$ \\
\hline 12 & $5 e(5-B r, B n)$ & 82 & $p$-Br-biphenyl & 12 & $61,61^{\prime}$ & 34 & $2.3: 1$ \\
\hline 13 & $\mathbf{5 f}(6-\mathrm{Cl}, \mathrm{Bn})$ & 25 & $p$-Br-biphenyl & 12 & $6 \mathrm{~m}, 6 \mathrm{~m}^{\prime}$ & 72 & $1.5: 1$ \\
\hline 14 & $5 \mathrm{~g}\left(7-\mathrm{CF}_{3}, \mathrm{Bn}\right)$ & 75 & $p$-Br-biphenyl & 12 & $6 n, 6 n^{\prime}$ & 47 & $1.4: 1$ \\
\hline
\end{tabular}

${ }^{a}$ Compounds 5 were prepared on a $0.3 \mathrm{mmol}$ scale, starting from the proper $\mathrm{R}^{1}, \mathrm{R}^{2}$-substituted isatin (1.0 equiv.), tert-butyl carbazate (1 equiv.), in ethanol $(0.3 \mathrm{M})$, at reflux for two hours, to give the corresponding N-Boc hydrazone intermediates 4 . Allylation reactions were conducted on 4 (1 equiv.), allyl bromide ( 2 equiv.) and metal powder ( 2 equiv.), in methanol $(0.1 \mathrm{M})$, at $40{ }^{\circ} \mathrm{C}$ for 3 hours. Carboamination reactions were carried out on a $0.2 \mathrm{mmol}$ scale, with 1.0 equiv. of $5,1.3$ equiv. of aryl halide, 1.3 equiv. of $\mathrm{NaO}{ }^{t} \mathrm{Bu}, 4 \mathrm{~mol} \%$ of $\mathrm{Pd}_{2}\left(\mathrm{dba}_{3}, 4 \mathrm{~mol} \%\right.$ of $\mathrm{XPhos}$, toluene $(0.2 \mathrm{M}) .{ }^{b}$ Isolated yields. ${ }^{c}$ Sum of the isolated yields for each diastereoisomer. ${ }^{d}$ Determined by ${ }^{1} \mathrm{H}$ NMR of the crude reaction product. ${ }^{e}$ No reaction. 


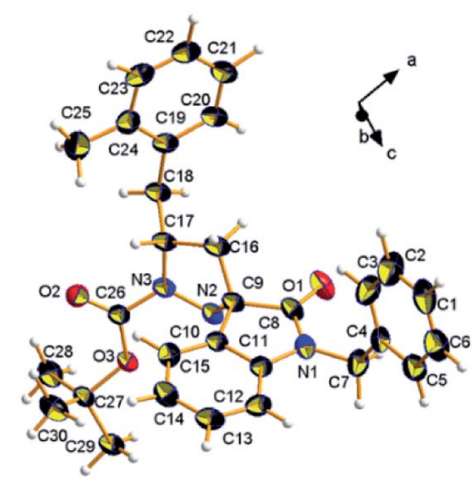

Fig. 3 ORTEP view of compound $6 f^{\prime}$ at room temperature, with the atom-numbering scheme and the crystallographic reference system highlighted. Thermal ellipsoids of non- $\mathrm{H}$ atoms were drawn at the $30 \%$ probability level.

catalysed reaction, probably due to the instability of the nitro group in basic conditions.

In order to further confirm the relative stereochemistry of the two diastereoisomeric series of products $\mathbf{6}$, as determined by NMR, a single crystal of the diastereoisomer $\mathbf{6} \mathbf{f}^{\prime}$ was subjected to single-crystal X-ray diffraction analysis. The experiment unambiguously assigns the $3^{\prime} S^{*}, 5^{\prime} R^{*}$ (cis) relative configuration, highlighting at the same time the preferred solid-state conformation (Fig. 3. For a full discussion of the crystallographic results, see the ESI $\dagger$ ).

\section{Conclusion}

We developed an efficient strategy for the synthesis of a novel class of oxindole-based spiro compounds, bearing the unusual pyrazolidine ring at the $\mathrm{C} 3$ stereogenic center. The reactivity of 3hydrazonoindolin-2-ones under various allylation conditions was explored, developing a robust protocol for the multi-gram conversion of variously substituted isatins into the corresponding N-Boc allyl hydrazine derivatives. Through a screening of the carboamination reaction conditions, it was possible to react effectively such intermediates with a variety of aryl halides, affording the cyclized spiro compounds in satisfactory yields. Both cis and trans diastereoisomers, with respect to the pyrazolidine ring, were obtained. Further work is currently underway, aimed at establishing spirooxindole-fused pyrazolidines as possible lead compounds for drug discovery programs.

\section{Experimental section}

\section{General procedures}

All commercial materials (Aldrich, Fluorochem) were used without further purification. All solvents were of reagent grade or HPLC grade. Reactions requiring anhydrous conditions were performed under nitrogen atmosphere. All reactions were monitored by thin layer chromatography (TLC) on precoated silica gel Merck 60 F254; spots were visualized with UV light $(254 \mathrm{~nm})$ or by treatment with $\mathrm{KMnO}_{4}$ solution in water or ninhydrin solution in ethanol. Products were purified by flash chromatography (FC) on silica gel 60 (230-400 mesh). Yields refer to isolated compounds estimated to be $>95 \%$ pure as determined by ${ }^{1} \mathrm{H}$ NMR. NMR spectra were recorded on Bruker 300 or Advance 400 spectrometers, using TMS as an internal standard. ${ }^{13} \mathrm{C}$ NMR spectra have been recorded using the APT pulse sequence. Chemical shifts are reported in parts per million relative to the residual solvent, coupling constants $(J)$ are given in Hz. Multiplicities in ${ }^{1} \mathrm{H}$ NMR are reported as follows: $\mathrm{s}=$ singlet, $\mathrm{d}=$ doublet, $\mathrm{t}=$ triplet, $\mathrm{m}=$ multiplet, $\mathrm{br} \mathrm{s}$ = broad singlet. High-resolution MS spectra (HRMS) were recorded with a Thermo Fisher LCQ Fleet ion trap mass spectrometer, equipped with an ESI source.

\section{Typical procedure (TP-A) for the synthesis of $N$-Boc hydrazone derivatives $(\mathbf{4 a}-\mathbf{4} \mathbf{j})$}

A mixture of $\mathrm{R}^{1}, \mathrm{R}^{2}$-substituted isatin $(0.3 \mathrm{mmol})$ and tert-butyl carbazate $(0.3 \mathrm{mmol})$ were suspended in ethanol $(0.3 \mathrm{M})$ and the reaction was stirred at reflux for 2 hours. The reaction was cooled down to room temperature, and then the solid was filtered and washed with cold ethanol. The pure product was obtained without need of purification.

\section{Typical procedure (TP-B) for the synthesis of $\mathrm{N}$-Boc hydrazine} derivatives $(5 \mathbf{a}-5 \mathbf{j})$

To a suspension of $N$-Boc hydrazone derivative $(0.3 \mathrm{mmol})$ in methanol $(0.1 \mathrm{M})$, indium $(0.6 \mathrm{mmol})$ and allyl bromide $(0.6$ $\mathrm{mmol}$ ) were added. After stirring at $40{ }^{\circ} \mathrm{C}$ for three hours, saturated aqueous $\mathrm{NH}_{4} \mathrm{Cl}$ solution was added and the mixture was extracted twice with ethyl acetate. The combined organic layer was washed with brine, dried over anhydrous $\mathrm{Na}_{2} \mathrm{SO}_{4}$, filtered and concentrated in vacuo to afford the crude product, which was purified by FC as indicated below.

\section{Typical procedure (TP-C) for the synthesis of 3-spiro-Pyrazolidyl- Oxindoles derivatives $\left(6 \mathbf{6}, 6 \mathbf{a}^{\prime}-6 \mathbf{n}, 6 \mathbf{n}^{\prime}\right)$}

The N-Boc hydrazine derivative $(0.2 \mathrm{mmol})$, the aryl halide $(0.26$ $\mathrm{mmol})$, sodium tert-butoxide $(0.26 \mathrm{mmol}), \mathrm{Pd}_{2}(\mathrm{dba})_{3}(4 \mathrm{~mol} \%)$ and XPhos (4 mol\%) were suspended in anhydrous toluene $(0.2$ $\mathrm{M})$ and the reaction was stirred at $90^{\circ} \mathrm{C}$. After the completion of reaction (monitored by TLC), saturated aqueous $\mathrm{NH}_{4} \mathrm{Cl}$ solution was added and the reaction was extracted twice with ethyl acetate. The combined organic layer was then dried over anhydrous $\mathrm{Na}_{2} \mathrm{SO}_{4}$, filtered and concentrated in vacuo to afford the crude product. This was purified by FC (hexane : ethyl acetate, as described below), affording the desired product, as separated $\left(3^{\prime}-5^{\prime}\right.$-trans) and $\left(3^{\prime}-5^{\prime}-\right.$ cis $)$ diastereoisomers (except for 61, 6l').

Analytical data of products $\left(4 a-4 j, 5 a-5 i, 6 a, 6 a^{\prime}-6 n, 6 n^{\prime}, 3 b, 7-\right.$ 13)

(Z)-tert-Butyl 2-(1-benzyl-2-oxoindolin-3-ylidene) hydrazine carboxylate (4a). Prepared according to TP-A, starting from $\mathrm{N}$ benzyl isatin. Yellow solid (96\% yield). ${ }^{1} \mathrm{H}$ NMR $(300 \mathrm{MHz}$, $\left.\mathrm{CDCl}_{3}\right) \delta 12.32$ (m, br, 1H), 7.74 (d, br, $\left.J=7.8 \mathrm{~Hz}, 1 \mathrm{H}\right), 7.37-7.20$ 
(m, 6H), $7.08(\mathrm{t}, J=7.8 \mathrm{~Hz}, 1 \mathrm{H}), 6.78(\mathrm{~d}, J=7.8 \mathrm{~Hz}, 1 \mathrm{H}), 4.94(\mathrm{~s}$, 2H), 1.56 (s, 9H). ${ }^{13} \mathrm{C}$ NMR (100 MHz, $\left.\mathrm{CDCl}_{3}\right) \delta$ 161.6, 152.3, 142.1, 135.1, 133.8 (br), 130.6, 129.0 (2C), 128.0, 127.4 (2C), 123.4, 121.3, 120.2 , 109.7, 82.6, 43.4, 28.2 (3C). HRMS (ESI) $m / z$ : $352.1661[\mathrm{M}+\mathrm{H}]^{+}$; calcd for $\mathrm{C}_{20} \mathrm{H}_{22} \mathrm{~N}_{3} \mathrm{O}_{3}{ }^{+}, 352.1656$.

tert-Butyl 2-(1-methyl-2-oxoindolin-3-ylidene)hydrazinecarboxylate (4b). Prepared according to TP-A, starting from $N$-methyl isatin. Light yellow solid (72\% yield). ${ }^{1} \mathrm{H} \mathrm{NMR} \mathrm{(300} \mathrm{MHz}, \mathrm{CDCl}_{3}$, $1.5: 1 Z: E$ mixture) $\delta 12.27$ (m, br, 0.6H), $8.79(\mathrm{~m}, \mathrm{br}, 0.4 \mathrm{H}), 7.71$ (d, br, $J=7.8 \mathrm{~Hz}, 0.6 \mathrm{H}), 7.56(\mathrm{~d}, \mathrm{br}, J=7.8 \mathrm{~Hz}, 0.4 \mathrm{H}), 7.42(\mathrm{t}, J=$ $7.8 \mathrm{~Hz}, 0.4 \mathrm{H}), 7.35(\mathrm{t}, J=7.8 \mathrm{~Hz}, 0.6 \mathrm{H}), 7.10(\mathrm{t}, J=7.8 \mathrm{~Hz}, 1 \mathrm{H})$, $6.90(\mathrm{~d}, J=7.8 \mathrm{~Hz}, 0.4 \mathrm{H}), 6.85(\mathrm{~d}, J=7.8 \mathrm{~Hz}, 0.6 \mathrm{H}), 3.25(\mathrm{~s}, 1.8 \mathrm{H})$, 3.24 (s, 1.2H), 1.57 (s, 3.6H), 1.55 (s, 5.4H). ${ }^{13} \mathrm{C}$ NMR (100 MHz, $\mathrm{CDCl}_{3}, 1.5: 1 \mathrm{Z}: E$ mixture) $\delta 160.8$ and 160.7 (1C), 155.6 and 155.2 (1C), 152.4, 145.6 and 135.1 (1C), 136.0 and 116.2 (1C), 133.0 and $131.3(1 \mathrm{C}), 124.6$ and $123.8(1 \mathrm{C}), 123.3$ and $121.8(1 \mathrm{C}), 110.0$ and 109.4 (1C), 84.2 and 83.8 (1C), 29.0 and 28.8 (3C), 26.9. HRMS (ESI) $m / z: 276.1349[\mathrm{M}+\mathrm{H}]^{+}$; calcd for $\mathrm{C}_{14} \mathrm{H}_{18} \mathrm{~N}_{3} \mathrm{O}_{3}{ }^{+}, 276.1343$.

(Z)-tert-Butyl 2-(1-((E)-3-(3,4-dichlorophenyl)allyl)-2-oxoindolin -3-ylidene)hydrazinecarboxylate (4c). Prepared according to TP-A, starting from $N$-((3,4-dichlorophenyl)allyl) isatin. Yellow solid (93\% yield). ${ }^{1} \mathrm{H}$ NMR (300 MHz, $\mathrm{CDCl}_{3}$ ) $\delta 12.25(\mathrm{~m}, \mathrm{br}, 1 \mathrm{H}), 7.76$ $(\mathrm{d}, J=7.8 \mathrm{~Hz}, 1 \mathrm{H}), 7.45-7.28$ (m, 3H), 7.19-7.07 (m, 2H), 6.87 (d, $J$ $=7.8 \mathrm{~Hz}, 1 \mathrm{H}), 6.48(\mathrm{~d}, J=15.6 \mathrm{~Hz}, 1 \mathrm{H}), 6.20(\mathrm{dt}, J=15.6$ and $5.8 \mathrm{~Hz}, 1 \mathrm{H}), 4.42(\mathrm{~d}, J=5.8 \mathrm{~Hz}, 2 \mathrm{H}), 1.55(\mathrm{~s}, 9 \mathrm{H}) .{ }^{13} \mathrm{C}$ NMR $(100$ $\left.\mathrm{MHz}, \mathrm{CDCl}_{3}\right) \delta 161.2,155.1,152.2,141.9,136.0,132.8,132.3$, 131.8, 131.0, 130.7, 128.3, 125.7, 124.2, 123.5, 121.4, 120.2, 109.3, 82.7, 41.3, 28.1 (3C). HRMS (ESI) $m / z: 446.1027[\mathrm{M}+\mathrm{H}]^{+}$; calcd for $\mathrm{C}_{22} \mathrm{H}_{22} \mathrm{~N}_{3} \mathrm{O}_{3}{ }^{+}, 446.1033$.

tert-Butyl 2-(1-benzyl-5-methyl-2-oxoindolin-3-ylidene)hydrazinecarboxylate (4d). Prepared according to TP-A, starting from $\mathrm{N}$ benzyl, 5-methyl isatin. Yellow solid (70\% yield). ${ }^{1} \mathrm{H}$ NMR (300 $\mathrm{MHz}, \mathrm{CDCl}_{3}, 7: 3$ tautomer mixture) $\delta 12.29(\mathrm{~m}, \mathrm{br}, 0.7 \mathrm{H}), 8.05(\mathrm{~s}$, br, 0.3H), 7.57 (s, br, 0.7H), 7.37-7.23 (m, 3.7H), 7.16-7.00 (m, 2H), $6.84(\mathrm{~d}, \mathrm{br}, J=7.8 \mathrm{~Hz}, 0.3 \mathrm{H}), 6.65(\mathrm{~d}, J=7.8 \mathrm{~Hz}, 0.7 \mathrm{H}), 6.50(\mathrm{~d}, J=$ $7.8 \mathrm{~Hz}, 0.3 \mathrm{H}), 4.91(\mathrm{~s}, 1.4 \mathrm{H}), 4.70(\mathrm{~s}, \mathrm{br}, 0.6 \mathrm{H}), 2.40-1.89(\mathrm{~m}, \mathrm{br}$, $0.3 \mathrm{H}), 2.29(\mathrm{~s}, 3 \mathrm{H}), 1.55(\mathrm{~s}, 6.3 \mathrm{H}), 1.51(\mathrm{~s}, 2.7 \mathrm{H}) .{ }^{13} \mathrm{C}$ NMR $(100 \mathrm{MHz}$, $\left.\mathrm{CDCl}_{3}\right) \delta 168.4$ and $161.6(1 \mathrm{C}), 168.3$ and $154.9(1 \mathrm{C}), 152.4$ and 58.2 (1C), 139.9 and 137.9 (1C), 136.7 and 135.2 (1C), 133.1 and 131.9 (1C), 131.9-121.9 (7C), 120.0 and 118.2 (1C), 109.4 and 107.9 (1C), 82.6 and 78.6 (1C), 43.4 and 43.1 (1C), 28.6 and 28.2 (3C), 21.1 and 21.0 (1C). HRMS (ESI) $m / z: 366.1804[\mathrm{M}+\mathrm{H}]^{+}$; calcd for $\mathrm{C}_{21} \mathrm{H}_{24} \mathrm{~N}_{3} \mathrm{O}_{3}{ }^{+}, 366.1812$.

(Z)-tert-Butyl 2-(1-benzyl-5-bromo-2-oxoindolin-3-ylidene)hydrazinecarboxylate (4e). Prepared according to TP-A, starting from $\mathrm{N}$ benzyl, 5-bromo isatin. Yellow solid (84\% yield). ${ }^{1} \mathrm{H}$ NMR (300 $\left.\mathrm{MHz}, \mathrm{CDCl}_{3}\right) \delta 12.23(\mathrm{~m}, \mathrm{br}, 1 \mathrm{H}), 7.87$ (d, br, $\left.J=2.0 \mathrm{~Hz}, 1 \mathrm{H}\right), 7.40-$ $7.22(\mathrm{~m}, 6 \mathrm{H}), 6.64(\mathrm{~d}, J=7.8 \mathrm{~Hz}, 1 \mathrm{H}), 4.92(\mathrm{~s}, 2 \mathrm{H}), 1.56(\mathrm{~s}, 9 \mathrm{H}) .{ }^{13} \mathrm{C}$ NMR $\left(100 \mathrm{MHz}, \mathrm{CDCl}_{3}\right) \delta 163.4,154.7,151.7,1342.8,135.0,134.6$, 133.5, 128.9 (2C), 128.0, 127.4 (2C), 126.8, 117.1, 111.7, 84.1, 43.9, 28.1 (3C). HRMS (ESI) $m / z: 430.0765[\mathrm{M}+\mathrm{H}]^{+}$; calcd for $\mathrm{C}_{20} \mathrm{H}_{21} \mathrm{BrN}_{3} \mathrm{O}_{3}{ }^{+}, 430.0761$.

(Z)-tert-Butyl 2-(1-benzyl-6-chloro-2-oxoindolin-3-ylidene)hydrazinecarboxylate (4f). Prepared according to TP-A, starting from $\mathrm{N}$ benzyl, 6-chloro isatin. Yellow solid (93\% yield). ${ }^{1} \mathrm{H}$ NMR (400 $\left.\mathrm{MHz} \mathrm{CDCl}_{3}\right) \delta 12.26(\mathrm{~m}, \mathrm{br}, 1 \mathrm{H}), 7.68(\mathrm{~d}, J=7.8 \mathrm{~Hz}, 1 \mathrm{H}), 7.42-7.27$ (m, 5H), 7.09 (dd, br, $J=7.8$ and $1.9 \mathrm{~Hz}, 1 \mathrm{H}), 6.80(\mathrm{~d}, J=1.9 \mathrm{~Hz}$,
1H), $4.94(\mathrm{~s}, 2 \mathrm{H}), 1.59(\mathrm{~s}, 9 \mathrm{H}) .{ }^{13} \mathrm{C} \mathrm{NMR}\left(100 \mathrm{MHz}, \mathrm{CDCl}_{3}\right) \delta 162.2$, 155.6, 152.7, 143.7, 137.0, 135.3, 129.8 (2C), 128.9, 128.0 (2C), 124.2, 122.8, 119.3, 111.0, 83.5, 44.3, 28.8 (3C). HRMS (ESI) $m / z$ : $386.1259[\mathrm{M}+\mathrm{H}]^{+}$; calcd for $\mathrm{C}_{20} \mathrm{H}_{21} \mathrm{ClN}_{3} \mathrm{O}_{3}{ }^{+}$, 386.1266.

(Z)-tert-Butyl 2-(1-benzyl-2-oxo-7-(trifluoromethyl)indolin-3 -ylidene)hydrazinecarboxylate (4g). Prepared according to TPA, starting from $N$-benzyl, 7-trifluoromethyl isatin. Yellow solid (64\% yield). ${ }^{1} \mathrm{H}$ NMR (300 MHz, $\left.\mathrm{CDCl}_{3}\right) \delta 12.23(\mathrm{~m}, \mathrm{br}$, $1 \mathrm{H}), 8.01$ (d, br, $J=7.6 \mathrm{~Hz}, 1 \mathrm{H}), 7.63$ (d, br, $J=8.6 \mathrm{~Hz}, 1 \mathrm{H})$, 7.34-7.17 (m, 4H), 7.10 (d, br, $J=6.9 \mathrm{~Hz}, 2 \mathrm{H}), 5.23(\mathrm{~s}, 2 \mathrm{H}), 1.54$ (s, 9H). ${ }^{13} \mathrm{C}$ NMR $\left(100 \mathrm{MHz}, \mathrm{CDCl}_{3}\right) \delta 162.4,156.7,151.9,139.6$, 135.7, 128.6 (2C), 128.3 (q, $J=7.4 \mathrm{~Hz}), 127.3,125.7$ (2C), 124.5, $122.9,123.1(\mathrm{q}, J=272.7 \mathrm{~Hz}), 122.8,113.8(\mathrm{q}, J=28.2 \mathrm{~Hz}), 83.1$, 45.6, 28.1 (3C). HRMS (ESI) $m / z: 420.1524[\mathrm{M}+\mathrm{H}]^{+}$; calcd for $\mathrm{C}_{21} \mathrm{H}_{21} \mathrm{~F}_{3} \mathrm{~N}_{3} \mathrm{O}_{3}{ }^{+}, 420.1530$.

(Z)-tert-Butyl 2-(1-benzyl-5-nitro-2-oxoindolin-3-ylidene)hydrazinecarboxylate (4h). Prepared according to TP-A, starting from $\mathrm{N}$ benzyl, 5-nitro isatin. Yellow solid (87\% yield). ${ }^{1} \mathrm{H}$ NMR $(400 \mathrm{MHz}$, $\left.\mathrm{CDCl}_{3}\right) \delta 12.22(\mathrm{~m}, \mathrm{br}, 1 \mathrm{H}), 8.66(\mathrm{~d}, J=1.8 \mathrm{~Hz}, 1 \mathrm{H}), 8.22(\mathrm{dd}, J=8.4$ and $1.9 \mathrm{~Hz}, 1 \mathrm{H}), 7.42-7.27(\mathrm{~m}, 5 \mathrm{H}), 6.91(\mathrm{~d}, J=8.4 \mathrm{~Hz}, 1 \mathrm{H}), 5.05(\mathrm{~s}$, $2 \mathrm{H}), 1.62$ (s, 9H). ${ }^{13} \mathrm{C} \mathrm{NMR}\left(100 \mathrm{MHz}, \mathrm{CDCl}_{3}\right) \delta 161.6,156.0,1151.6$, 146.2, 144.2, 134.1, 129.2 (2C), 128.5, 127.3 (2C), 126.4, 120.9, 116.8, 109.5, 83.5, 43.9, 28.1 (3C). HRMS (ESI) $m / z: 397.1500[\mathrm{M}+$ $\mathrm{H}]^{+}$; calcd for $\mathrm{C}_{20} \mathrm{H}_{21} \mathrm{~N}_{4} \mathrm{O}_{5}{ }^{+}, 397.1506$.

(Z)-tert-Butyl 2-(1-benzyl-5-methoxy-2-oxoindolin-3-ylidene) hydrazinecarboxylate (4i). Prepared according to TP-A, starting from $N$-benzyl, 5-methoxy isatin. Yellow solid (96\% yield). ${ }^{1} \mathrm{H}$ NMR $\left(400 \mathrm{MHz}, \mathrm{CDCl}_{3}\right) \delta 12.38(\mathrm{~m}, \mathrm{br}, 1 \mathrm{H}), 7.37-7.23(\mathrm{~m}, 6 \mathrm{H})$, $6.80(\mathrm{dd}, J=8.6$ and $2.9 \mathrm{~Hz}, 1 \mathrm{H}), 6.66(\mathrm{~d}, J=8.6 \mathrm{~Hz}, 1 \mathrm{H}), 4.90$ (s, 2H), $3.76(\mathrm{~s}, 3 \mathrm{H}), 1.58(\mathrm{~s}, 9 \mathrm{H}) .{ }^{13} \mathrm{C} \mathrm{NMR}\left(75 \mathrm{MHz}, \mathrm{CDCl}_{3}\right.$ ) $\delta 161.5,156.4,155.6,152.3,135.8,135.2,130.9,129.0$ (2C), 128.0, 127.4 (2C), 117.4, 110.6, 106.0, 82.6, 55.9, 43.4, 28.2 (3C). HRMS (ESI) $m / z: 382.1767[\mathrm{M}+\mathrm{H}]^{+}$; calcd for $\mathrm{C}_{21} \mathrm{H}_{24} \mathrm{~N}_{3} \mathrm{O}_{4}{ }^{+}$, 382.1761 .

(Z)-tert-Butyl 2-(1-benzyl-4-chloro-2-oxoindolin-3-ylidene)hydrazinecarboxylate (4j). Prepared according to TP-A, starting from $\mathrm{N}$ benzyl, 4-chloro isatin. Yellow solid (84\% yield). ${ }^{1} \mathrm{H}$ NMR (400 $\left.\mathrm{MHz}, \mathrm{CDCl}_{3}\right) \delta 12.49(\mathrm{~m}, \mathrm{br}, 1 \mathrm{H}), 7.41-7.25(\mathrm{~m}, 5 \mathrm{H}), 7.17(t, \mathrm{br}, J=$ $7.8 \mathrm{~Hz}, 1 \mathrm{H}), 7.08(\mathrm{~d}, J=7.8 \mathrm{~Hz}, 1 \mathrm{H}), 6.71(\mathrm{~d}, J=7.8 \mathrm{~Hz}, 1 \mathrm{H}), 4.97(\mathrm{~s}$, $2 \mathrm{H}), 1.60(\mathrm{~s}, 9 \mathrm{H}) .{ }^{13} \mathrm{C} \mathrm{NMR}\left(100 \mathrm{MHz}, \mathrm{CDCl}_{3}\right) \delta 161.0,154.8,151.8$, 143.1, 134.8, 130.7, 129.5, 129.0 (2C), 128.1, 127.3 (2C), 125.0, 117.2, 108.0, 82.7, 43.6, 28.2 (3C). HRMS (ESI) $m / z: 386.1261[\mathrm{M}+$ $\mathrm{H}]^{+}$; calcd for $\mathrm{C}_{20} \mathrm{H}_{21} \mathrm{ClN}_{3} \mathrm{O}_{3}{ }^{+}, 386.1266$.

tert-Butyl 2-(3-allyl-1-benzyl-2-oxoindolin-3-yl)hydrazinecarbox ylate (5a). Prepared according to TP-B starting from compound 4a, and purified by FC (hexane : ethyl acetate from $7: 3$ to $3: 7$ ). Yellow foam (94\% yield). ${ }^{1} \mathrm{H} \mathrm{NMR}\left(400 \mathrm{MHz}, \mathrm{CDCl}_{3}\right) \delta 7.46(\mathrm{~d}, \mathrm{br}, J$ $=7.2 \mathrm{~Hz}, 1 \mathrm{H}), 7.34-7.22(\mathrm{~m}, 5 \mathrm{H}), 7.17(t, \mathrm{br}, J=7.5 \mathrm{~Hz}, 1 \mathrm{H}), 7.04$ $(t, \mathrm{br}, J=7.5 \mathrm{~Hz}, 1 \mathrm{H}), 6.85(\mathrm{~d}, J=7.8 \mathrm{~Hz}, 1 \mathrm{H}), 6.08(\mathrm{~m}, \mathrm{br}, 1 \mathrm{H})$, $5.52(\mathrm{~m}, 1 \mathrm{H}), 5.09$ (d, br, $J=17.0 \mathrm{~Hz}, 1 \mathrm{H}), 5.00$ (d, br, $J=10.2 \mathrm{~Hz}$, $1 \mathrm{H}), 4.94$ (d, br, $J=16.0 \mathrm{~Hz}, 1 \mathrm{H}), 4.87$ (d, br, $J=16.0 \mathrm{~Hz}, 1 \mathrm{H}$ ), 4.81-4.47 (m, br, 1H), 2.73 (dd, br, $J=13.3$ and $6.2 \mathrm{~Hz}, 1 \mathrm{H}), 2.64$ (dd, br, $J=13.3$ and $8.5 \mathrm{~Hz}, 1 \mathrm{H}), 1.32$ (s, 9H). ${ }^{13} \mathrm{C}$ NMR $(100 \mathrm{MHz}$, $\left.\mathrm{CDCl}_{3}\right) \delta 177.3,156.0,143.2,135.6,130.7,129.2$, 128.7 (2C), 127.8, 127.6, 127.2 (2C), 125.2, 122.6, 120.1, 109.1, 80.5, 68.6, 43.8, 39.6, 28.1 (3C). HRMS (ESI) $m / z: 416.1938[\mathrm{M}+\mathrm{Na}]^{+}$; calcd for $\mathrm{C}_{23} \mathrm{H}_{27} \mathrm{~N}_{3} \mathrm{NaO}_{3}^{+}, 416.1945$. 
tert-Butyl 2-(3-allyl-1-methyl-2-oxoindolin-3-yl)hydrazinecarboxylate (5b). Prepared according to TP-B starting from compound $\mathbf{4 b}$, and purified by FC (hexane : ethyl acetate $7: 3)$. Yellow foam $(82 \%$ yield). ${ }^{1} \mathrm{H}$ NMR (300 MHz, $\left.\mathrm{CDCl}_{3}\right) \delta 7.45(\mathrm{~d}, J=7.8 \mathrm{~Hz}, 1 \mathrm{H}), 7.29(\mathrm{t}$, $J=7.8 \mathrm{~Hz}, 1 \mathrm{H}), 7.07(\mathrm{t}, J=7.8 \mathrm{~Hz}, 1 \mathrm{H}), 6.80(\mathrm{~d}, J=7.8 \mathrm{~Hz}, 1 \mathrm{H}), 5.98$ (m, br, 1H), 5.48 (m, 1H), 5.08-4.94 (m, 2H), 3.18 (s, 3H), 3.18-2.77 (m, br, 1H), 2.67 (dd, $J=15.6$ and $6.8 \mathrm{~Hz}, 1 \mathrm{H}), 2.55(\mathrm{dd}, J=15.6$ and $8.8 \mathrm{~Hz}, 1 \mathrm{H}), 1.31(\mathrm{~s}, 9 \mathrm{H}) .{ }^{13} \mathrm{C} \mathrm{NMR}\left(100 \mathrm{MHz}, \mathrm{CDCl}_{3}\right) \delta 177.3$, 156.1, 143.9, 130.6, 129.2, 127.7, 125.2, 122.5, 119.8, 107.9, 80.3, 68.6, 39.5, 28.1 (3C), 26.1. HRMS (ESI) $m / z: 340.1636[\mathrm{M}+\mathrm{Na}]^{+}$; calcd for $\mathrm{C}_{17} \mathrm{H}_{23} \mathrm{~N}_{3} \mathrm{NaO}_{3}{ }^{+}, 340.1632$.

(E)-tert-Butyl 2-(3-allyl-1-(3-(3,4-dichlorophenyl)allyl)-2-oxoindolin-3-yl)hydrazinecarboxylate (5c). Prepared according to TP-B starting from compound $\mathbf{4 c}$, and purified by FC (hexane : ethyl acetate 1 : 1). Dark yellow foam (93\% yield). ${ }^{1} \mathrm{H}$ NMR $(300 \mathrm{MHz}$, $\left.\mathrm{CDCl}_{3}\right) \delta 7.47(\mathrm{~d}, \mathrm{br}, J=7.8 \mathrm{~Hz}, 1 \mathrm{H}), 7.42-7.19(\mathrm{~m}, 3 \mathrm{H}), 7.17-7.03$ $(\mathrm{m}, 2 \mathrm{H}), 6.79$ (d, br, $J=7.8 \mathrm{~Hz}, 1 \mathrm{H}), 6.41(\mathrm{~d}, \mathrm{br}, J=15.6 \mathrm{~Hz}, 1 \mathrm{H})$, $6.16(\mathrm{dt}, \mathrm{br}, J=15.6$ and $4.9 \mathrm{~Hz}, 1 \mathrm{H}), 5.98(\mathrm{~m}, \mathrm{br}, 1 \mathrm{H}), 5.50(\mathrm{~m}, \mathrm{br}$, $1 \mathrm{H}), 5.07$ (d, $J=17.6 \mathrm{~Hz}, 1 \mathrm{H}), 5.01(\mathrm{~d}, J=10.8 \mathrm{~Hz}, 1 \mathrm{H}), 5.46(\mathrm{~d}, \mathrm{br}$, $J=4.9 \mathrm{~Hz}, 2 \mathrm{H}), 2.81-2.50(\mathrm{~m}, \mathrm{br}, 1 \mathrm{H}), 2.71(\mathrm{dd}, J=13.7$ and $6.8 \mathrm{~Hz}, 1 \mathrm{H}), 2.60(\mathrm{dd}, J=13.7$ and $8.8 \mathrm{~Hz}, 1 \mathrm{H}), 1.30(\mathrm{~s}, 9 \mathrm{H}) .{ }^{13} \mathrm{C}$ NMR (100 MHz, $\mathrm{CDCl}_{3}$ ) $\delta 177.0,156.2,143.0,136.4,132.7,131.5$, 130.7, 130.4, 129.9, 129.3, 128.2, 127.8, 125.6, 125.4, 124.9, 122.7, 120.1, 108.7, 80.5, 68.6, 41.5, 39.7, 28.1 (3C). HRMS (ESI) $m / z$ : $510.1028[\mathrm{M}+\mathrm{Na}]^{+}$; calcd for $\mathrm{C}_{25} \mathrm{H}_{27} \mathrm{Cl}_{2} \mathrm{~N}_{3} \mathrm{NaO}_{3}{ }^{+}, 510.1322$.

tert-Butyl 2-(3-allyl-1-benzyl-5-methyl-2-oxoindolin-3-yl)hydrazinecarboxylate (5d). Prepared according to TP-B starting from compound 4d, and purified by FC (hexane : ethyl acetate $7: 3$ ). Yellow foam (98\% yield). ${ }^{1} \mathrm{H}$ NMR $\left(300 \mathrm{MHz}, \mathrm{CDCl}_{3}\right) \delta 7.33-7.20$ (m, 6H), 6.95 (d, br, $J=7.8 \mathrm{~Hz}, 1 \mathrm{H}), 6.53$ (d, br, $J=7.8 \mathrm{~Hz}, 1 \mathrm{H})$, 5.98 (m, br, 1H), 5.50 (m, 1H), 5.08 (d, br, $J=17.6 \mathrm{~Hz}, 1 \mathrm{H}), 4.99$ (d, br, $J=10.7 \mathrm{~Hz}, 1 \mathrm{H}), 4.92$ (d, br, $J=15.6 \mathrm{~Hz}, 1 \mathrm{H}), 4.81$ (d, br, $J=$ $15.6 \mathrm{~Hz}, 1 \mathrm{H}), 3.72(\mathrm{~m}, \mathrm{br}, 1 \mathrm{H}), 2.71$ (dd, $J=12.7$ and $5.8 \mathrm{~Hz}, 1 \mathrm{H}$ ), $2.62(\mathrm{dd}, J=12.7$ and $8.8 \mathrm{~Hz}, 1 \mathrm{H}), 2.29(\mathrm{~s}, 3 \mathrm{H}), 1.33(\mathrm{~s}, 9 \mathrm{H}) .{ }^{13} \mathrm{C}$ NMR (100 MHz, $\mathrm{CDCl}_{3}$ ) $\delta 173.3,156.0,140.8,135.7,132.0,130.8$, 129.4, 128.7 (2C), 127.8, 127.5, 127.2 (2C), 125.9, 120.0, 108.9, 80.5, 68.6, 43.8, 39.6, 28.1 (3C), 21.1. HRMS (ESI) $m / z: 430.2096[\mathrm{M}+$ $\mathrm{Na}]^{+}$; calcd for $\mathrm{C}_{24} \mathrm{H}_{29} \mathrm{~N}_{3} \mathrm{NaO}_{3}{ }^{+}, 430.2101$.

tert-Butyl 2-(3-allyl-1-benzyl-5-bromo-2-oxoindolin-3-yl)hydrazinecarboxylate (5e). Prepared according to TP-B starting from compound 4e, and purified by FC (hexane : ethyl acetate $7: 3$ ). Yellow foam (82\% yield). ${ }^{1} \mathrm{H}$ NMR (300 MHz, $\left.\mathrm{CDCl}_{3}\right) \delta 7.57$ (d, $J$ $=1.8 \mathrm{~Hz}, 1 \mathrm{H}), 7.35-7.17(\mathrm{~m}, 6 \mathrm{H}), 6.51(\mathrm{~d}, J=7.8 \mathrm{~Hz}, 1 \mathrm{H}), 5.91$ $(\mathrm{m}, \mathrm{br}, 1 \mathrm{H}), 5.50(\mathrm{~m}, 1 \mathrm{H}), 5.10(\mathrm{~d}, \mathrm{br}, J=16.6 \mathrm{~Hz}, 1 \mathrm{H}), 5.03(\mathrm{~d}, \mathrm{br}$, $J=9.8 \mathrm{~Hz}, 1 \mathrm{H}), 4.91(\mathrm{~d}, \mathrm{br}, J=15.0 \mathrm{~Hz}, 1 \mathrm{H}), 4.83$ (d, br, $J=$ $15.0 \mathrm{~Hz}, 1 \mathrm{H}), 3.04-2.71(\mathrm{~m}, \mathrm{br}, 1 \mathrm{H}), 2.69(\mathrm{dd}, J=13.7$ and $6.8 \mathrm{~Hz}$, $1 \mathrm{H}), 2.61$ (dd, $J=13.7$ and $8.8 \mathrm{~Hz}, 1 \mathrm{H}), 1.34(\mathrm{~s}, 9 \mathrm{H}) .{ }^{13} \mathrm{C} \mathrm{NMR}$ $\left(100 \mathrm{MHz}, \mathrm{CDCl}_{3}\right) \delta 176.8,156.2,142.3,135.1,131.9,130.2$, 130.1, 128.8 (2C), 128.5, 127.7, 127.1 (2C), 120.5, 115.3, 110.6, 80.6, 69,1, 43.9, 39.6, 28.1 (3C). HRMS (ESI) m/z: 494.1055 [M + $\mathrm{Na}]^{+}$; calcd for $\mathrm{C}_{23} \mathrm{H}_{26} \mathrm{BrN}_{3} \mathrm{NaO}_{3}{ }^{+}, 494.1050$.

tert-Butyl 2-(3-allyl-1-benzyl-6-chloro-2-oxoindolin-3-yl)hydrazinecarboxylate (5f). Prepared according to TP-B starting from compound $\mathbf{4 f}$, and purified by FC (hexane : ethyl acetate from $8: 2$ to $4: 6)$. Dark yellow foam (25\% yield). ${ }^{1} \mathrm{H}$ NMR (300 MHz, $\left.\mathrm{CDCl}_{3}\right) \delta 7.42-7.20(\mathrm{~m}, 6 \mathrm{H}), 7.01(\mathrm{~d}, \mathrm{br}, J=7.8 \mathrm{~Hz}, 1 \mathrm{H}), 6.33(\mathrm{~m}$,
1H), $5.92(\mathrm{~m}, \mathrm{br}, 1 \mathrm{H}), 5.50(\mathrm{~m}, 1 \mathrm{H}), 5.07$ (d, br, $J=16.6 \mathrm{~Hz}, 1 \mathrm{H})$, $5.01(\mathrm{~d}, \mathrm{br}, J=9.8 \mathrm{~Hz}, 1 \mathrm{H}), 4.89$ (d, $J=15.6 \mathrm{~Hz}, 1 \mathrm{H}), 4.82(\mathrm{~d}, J=$ $15.6 \mathrm{~Hz}, 1 \mathrm{H}), 3.35-2.84(\mathrm{~m}, \mathrm{br}, 1 \mathrm{H}), 2.69$ (dd, $J=13.7$ and $6.8 \mathrm{~Hz}$, $1 \mathrm{H}), 2.60(\mathrm{dd}, J=13.7$ and $8.8 \mathrm{~Hz}, 1 \mathrm{H}), 1.32(\mathrm{~s}, 9 \mathrm{H}) .{ }^{13} \mathrm{C}$ NMR $(100$ $\left.\mathrm{MHz}, \mathrm{CDCl}_{3}\right) \delta 177.3,156.1,144.4,135.1,134.9,130.3,128.9$ (2C), 127.8, 127.1 (2C), 126.3, 126.2, 122.5, 120.5, 109.7, 80.6, 68.5, 43.9, 39.6, 28.1 (3C). HRMS (ESI) $m / z: 450.1560[\mathrm{M}+\mathrm{Na}]^{+}$; calcd for $\mathrm{C}_{23} \mathrm{H}_{26} \mathrm{ClN}_{3} \mathrm{NaO}_{3}^{+}, 450.1555$.

tert-Butyl 2-(3-allyl-1-benzyl-2-oxo-7-(trifluoromethyl)indolin -3-yl)hydrazinecarboxylate (5g). Prepared according to TP-B starting from compound $\mathbf{4 g}$, and purified by FC (hexane : ethyl acetate $7: 3)$. Yellow foam (75\% yield). ${ }^{1} \mathrm{H}$ NMR (300 $\left.\mathrm{MHz}, \mathrm{CDCl}_{3}\right) \delta 7.74(\mathrm{~d}, \mathrm{br}, J=6.9 \mathrm{~Hz}, 1 \mathrm{H}), 7.56(\mathrm{~d}, \mathrm{br}, J=7.9 \mathrm{~Hz}$, 1H), 7.33-7.07 (m, 6H), 5.91 (m, br, 1H), 5.48 (m, 1H), 5.20 (d, J $=16.6 \mathrm{~Hz}, 1 \mathrm{H}), 5.13(\mathrm{~d}, J=16.6 \mathrm{~Hz}, 1 \mathrm{H}), 5.06(\mathrm{~d}, \mathrm{br}, J=17.6 \mathrm{~Hz}$, $1 \mathrm{H}), 5.05$ (d, br, $J=10.8 \mathrm{~Hz}, 1 \mathrm{H}), 3.34$ (m, br, 1H), 2.74-2.56 (m, 2H), 1.32 (s, 9H). ${ }^{13} \mathrm{C}$ NMR (100 MHz, $\mathrm{CDCl}_{3}$ ) $\delta$ 178.8, 156.0, 141.5, 136.3, 130.9, 129.8, 129.1, $128.3(2 \mathrm{C}), 127.5$ (q, $J=5.9 \mathrm{~Hz})$, 126.9, 125.8 (2C), 123.3 (q, $J=271.3 \mathrm{~Hz}), 122.2,120.8,112.8$ (q, $J$ $=33.9 \mathrm{~Hz}$ ), 80.7, 67.0, 45.7, 40.0, 28.1 (3C). HRMS (ESI) $m / z$ : $484.1824[\mathrm{M}+\mathrm{Na}]^{+}$; calcd for $\mathrm{C}_{24} \mathrm{H}_{26} \mathrm{~F}_{3} \mathrm{~N}_{3} \mathrm{NaO}_{3}{ }^{+}, 484.1818$.

tert-Butyl 2-(3-allyl-1-benzyl-5-nitro-2-oxoindolin-3-yl)hydrazinecarboxylate (5h). Prepared according to TP-B starting from compound $\mathbf{4 h}$, and purified by FC (hexane : ethyl acetate $7: 3$ ). Yellow foam (71\% yield). ${ }^{1} \mathrm{H}$ NMR $\left(300 \mathrm{MHz}, \mathrm{CDCl}_{3}\right) \delta 8.32(\mathrm{~m}, \mathrm{br}$, $1 \mathrm{H}), 8.13$ (d, br, $J=8.7 \mathrm{~Hz}, 1 \mathrm{H}), 7.36-7.17(\mathrm{~m}, 5 \mathrm{H}), 6.71$ (d, $J=$ $8.7 \mathrm{~Hz}, 1 \mathrm{H}), 6.00(\mathrm{~m}, 1 \mathrm{H}), 5.49(\mathrm{~m}, 1 \mathrm{H}), 5.15-5.00(\mathrm{~m}, 2 \mathrm{H}), 4.97$ (d, $J=15.6 \mathrm{~Hz}, 1 \mathrm{H}), 4.91$ (d, $J=15.6 \mathrm{~Hz}, 1 \mathrm{H}), 3.54-2.87(\mathrm{~m}, \mathrm{br}, 1 \mathrm{H})$, $2.75(\mathrm{dd}, J=13.0$ and $5.8 \mathrm{~Hz}, 1 \mathrm{H}), 2.56(\mathrm{dd}, J=13.0$ and $7.8 \mathrm{~Hz}$, $1 \mathrm{H}), 1.31(\mathrm{~s}, 9 \mathrm{H}) .{ }^{13} \mathrm{C} \mathrm{NMR}\left(100 \mathrm{MHz}, \mathrm{CDCl}_{3}\right) \delta 177.4,156.4,148.9$, 143.4, 134.5, 129.6, 129.1, 129.0 (2C), 127.2 (2C), 126.2, 121.2, 121.0, 120.0, 108.8, 81.3, 68.7, 44.2, 39.6, 28.0 (3C). HRMS (ESI) m/ $z: 461.1791[\mathrm{M}+\mathrm{Na}]^{+}$; calcd for $\mathrm{C}_{23} \mathrm{H}_{26} \mathrm{~N}_{4} \mathrm{NaO}_{5}{ }^{+}, 461.1795$.

tert-Butyl 2-(3-allyl-1-benzyl-5-methoxy-2-oxoindolin-3-yl)hydrazinecarboxylate (5i). Prepared according to TP-B starting from compound $4 \mathbf{i}$, and purified by FC (hexane : ethyl acetate $7: 3$ ). Yellow foam (15\% yield). ${ }^{1} \mathrm{H}$ NMR (300 MHz, $\left.\mathrm{CDCl}_{3}\right) \delta 7.34-7.20$ $(\mathrm{m}, 5 \mathrm{H}), 7.09(\mathrm{~d}, \mathrm{br}, J=2.9 \mathrm{~Hz}, 1 \mathrm{H}), 6.69(\mathrm{dd}, J=8.7$ and $2.9 \mathrm{~Hz}$, $1 \mathrm{H}), 6.52(\mathrm{~d}, J=8.7 \mathrm{~Hz}, 1 \mathrm{H}), 6.00(\mathrm{~m}, \mathrm{br}, 1 \mathrm{H}), 5.51(\mathrm{~m}, 1 \mathrm{H}), 5.08$ (d, br, $J=17.6 \mathrm{~Hz}, 1 \mathrm{H}), 5.00(\mathrm{~d}, \mathrm{br}, J=11.7 \mathrm{~Hz}, 1 \mathrm{H}), 4.91$ (d, $J=$ $15.6 \mathrm{~Hz}, 1 \mathrm{H}), 4.82(\mathrm{~d}, J=15.6 \mathrm{~Hz}, 1 \mathrm{H}), 3.75(\mathrm{~s}, 3 \mathrm{H}), 2.71(\mathrm{dd}, J=$ 12.7 and $5.8 \mathrm{~Hz}, 1 \mathrm{H}), 2.62(\mathrm{dd}, J=12.7$ and $7.8 \mathrm{~Hz}, 1 \mathrm{H}), 2.52(\mathrm{~m}$, br, $1 \mathrm{H}), 1.32(\mathrm{~s}, 9 \mathrm{H}) .{ }^{13} \mathrm{C}$ NMR $\left(100 \mathrm{MHz}, \mathrm{CDCl}_{3}\right) \delta 177.8,156.7$ (2C), 137.1, 136.3, 131.3, 129.7, 129.4 (2C), 128.2, 127.9 (2C), 120.8, 114.8 (br), 112.8 (br), 110.2, 81.1, 69.6, 56.5, 44.5, 40.3, 28.8 (3C). HRMS (ESI) $m / z: 446.2056[\mathrm{M}+\mathrm{Na}]^{+}$; calcd for $\mathrm{C}_{24} \mathrm{H}_{29} \mathrm{~N}_{3} \mathrm{NaO}_{4}{ }^{+}, 446.2050$.

$\left(3 S^{*}, 5^{\prime} S^{*}\right)$-tert-Butyl $\quad 5^{\prime}$-([1,1'-biphenyl $]-4$-ylmethyl)-1-benzyl-2oxospiro[indoline-3, $3^{\prime}$-pyrazolidine]-1'-carboxylate $(6 a)$ and $\left(3 S^{*}\right.$ , $\left.5^{\prime} R^{*}\right)$-tert-butyl $5^{\prime}$-([1,1'-biphenyl]-4-ylmethyl)-1-benzyl-2-oxospiro [indoline-3, $\mathbf{3}^{\prime}$-pyrazolidine]-1' $\mathbf{1}^{\prime}$-carboxylate $\left(\mathbf{6 a}^{\prime}\right)$. Prepared according to TP-C starting from compound $\mathbf{5 a}$ and $p$-Br-biphenyl, and purified by FC (hexane : ethyl acetate $7: 3)$. Yellow foam $(98 \%$ yield, separated diastereoisomers, $1: 1 \mathrm{dr}$ ). Diastereoisomer 6a. ${ }^{1} \mathrm{H}$ NMR (400 MHz, $\left.\mathrm{CDCl}_{3}\right) \delta 7.65-7.57(\mathrm{~m}, 4 \mathrm{H}), 7.48(\mathrm{t}, \mathrm{br}, J=$ $7.7 \mathrm{~Hz}, 2 \mathrm{H}), 7.44-7.22(\mathrm{~m}, 9 \mathrm{H}), 7.16(\mathrm{t}, \mathrm{br}, J=7.8 \mathrm{~Hz}, 1 \mathrm{H}), 6.93(\mathrm{t}$, br, $J=7.8 \mathrm{~Hz}, 1 \mathrm{H}), 6.85$ (m, br, 1H), 6.65 (d, br, $J=7.8 \mathrm{~Hz}, 1 \mathrm{H})$, 
5.07-4.71 (m, br, 3H), $3.23(\mathrm{dd}, J=13.2$ and $3.8 \mathrm{~Hz}, 1 \mathrm{H}), 3.15$ (dd, $J=13.2$ and $7.0 \mathrm{~Hz}, 1 \mathrm{H}), 2.60(\mathrm{dd}, J=12.8$ and $8.2 \mathrm{~Hz}, 1 \mathrm{H}$ ), 2.25 (dd, $J=12.8$ and $7.9 \mathrm{~Hz}, 1 \mathrm{H}), 1.56$ (s, 9H). ${ }^{13} \mathrm{C}$ NMR (100 $\left.\mathrm{MHz}, \mathrm{CDCl}_{3}\right) \delta 177.2,156.7$ (br), 144.4, 141.5, 140.4, 137.5, 136.3, 131.1 (2C), 130.6, 129.5 (2C), 129.4 (2C), 128.3, 128.0 (3C), 127.9 (2C), 127.7 (2C), 126.2, 123.4, 123.2, 110.0, 81.4, 67.3, 61.0, 44.0 (2C), 41.1, 29.1 (3C). HRMS (ESI) $m / z: 568.2566[\mathrm{M}+\mathrm{Na}]^{+}$; calcd for $\mathrm{C}_{35} \mathrm{H}_{35} \mathrm{~N}_{3} \mathrm{NaO}_{5}{ }^{+}$, 568.2571. Diastereoisomer $6 \mathrm{a}^{\prime} .{ }^{1} \mathrm{H}$ NMR (400 MHz, $\left.\mathrm{CDCl}_{3}\right) \delta 7.62-7.53(\mathrm{~m}, 4 \mathrm{H}), 7.45(\mathrm{t}, \mathrm{br}, J=$ $7.7 \mathrm{~Hz}, 2 \mathrm{H}), 7.41-7.24(\mathrm{~m}, 9 \mathrm{H}), 7.17$ (t, br, $J=7.8 \mathrm{~Hz}, 1 \mathrm{H}), 7.05$ (d, br, $J=7.8 \mathrm{~Hz}, 1 \mathrm{H}), 7.00$ (t, br, $J=7.8 \mathrm{~Hz}, 1 \mathrm{H}), 6.74$ (d, br, $J=$ $7.8 \mathrm{~Hz}, 1 \mathrm{H}), 5.01$ (d, $J=15.6 \mathrm{~Hz}, 1 \mathrm{H}), 4.82(\mathrm{~d}, J=15.6 \mathrm{~Hz}, 1 \mathrm{H})$, $4.75(\mathrm{~m}, \mathrm{br}, 1 \mathrm{H}), 3.46(\mathrm{dd}, J=13.2$ and $4.4 \mathrm{~Hz}, 1 \mathrm{H}), 2.96(\mathrm{dd}, J=$ 13.2 and $9.0 \mathrm{~Hz}, 1 \mathrm{H}), 2.44(\mathrm{dd}, J=12.3$ and $8.5 \mathrm{~Hz}, 1 \mathrm{H}), 2.35$ (dd, $J=12.3$ and $7.3 \mathrm{~Hz}, 1 \mathrm{H}), 1.54(\mathrm{~s}, 9 \mathrm{H}) .{ }^{13} \mathrm{C} \mathrm{NMR}(100 \mathrm{MHz}$, $\left.\mathrm{CDCl}_{3}\right) \delta 174.8,156.4$ (br), 142.5, 141.6, 140.3, 137.7, 135.8, 132.3, 130.5 (2C), 129.6 (2C), 129.4 (2C), 128.5, 128.0 (4C), 127.9 (2C), 127.7 (2C), 124.0, 123.4, 110.4, 81.8, 68.7, 61.8, 45.4, 44.9, 41.6, 29.1 (3C). HRMS (ESI) $m / z: 568.2576[\mathrm{M}+\mathrm{Na}]^{+}$; calcd for $\mathrm{C}_{35} \mathrm{H}_{35} \mathrm{~N}_{3} \mathrm{NaO}_{5}{ }^{+}, 568.2571$.

$\left(3 S^{*}, 5^{\prime} S^{*}\right)$-tert-Butyl 1-benzyl-5' -(4-methoxybenzyl)-2-oxospiro [indoline-3, $3^{\prime}$-pyrazolidine]-1'-carboxylate (6b) and $\left(3 S^{*}, 5^{\prime} R^{*}\right)$ tert-butyl 1-benzyl-5' -(4-methoxybenzyl)-2-oxospiro[indoline-3, $3^{\prime}$ pyrazolidine]-1'-carboxylate $\left(\mathbf{6 b}^{\prime}\right)$. Prepared according to TP-C starting from compound $\mathbf{5 a}$ and $p$-Br-anisole, and purified by FC (hexane : ethyl acetate $7: 3$ ). Yellow foam (98\% yield, separated diastereoisomers, $1: 1 \mathrm{dr}$ ). Diastereoisomer $6 \mathrm{~b} .{ }^{1} \mathrm{H}$ NMR $\left(300 \mathrm{MHz}, \mathrm{CDCl}_{3}\right) \delta 7.33-7.18(\mathrm{~m}, 7 \mathrm{H}), 7.14(\mathrm{~d}, \mathrm{br}, J=7.8 \mathrm{~Hz}, 1 \mathrm{H})$, $6.92(\mathrm{t}, \mathrm{br}, J=7.8 \mathrm{~Hz}, 1 \mathrm{H}), 6.89(\mathrm{~d}, \mathrm{br}, J=8.8 \mathrm{~Hz}, 2 \mathrm{H}), 6.74(\mathrm{~d}, \mathrm{br}, J$ $=7.8 \mathrm{~Hz}, 1 \mathrm{H}), 6.62(\mathrm{~d}, J=7.8 \mathrm{~Hz}, 1 \mathrm{H}), 5.00-4.97(\mathrm{~m}, \mathrm{br}, 3 \mathrm{H}), 4.11$ (m, br, 1H), 3.81 (s, 3H), 3.11-2.97 (m, 2H), 2.51 (dd, $J=12.7$ and $7.8 \mathrm{~Hz}, 1 \mathrm{H}), 2.13$ (dd, $J=12.7$ and $7.8 \mathrm{~Hz}, 1 \mathrm{H}), 1.53(\mathrm{~s}, 9 \mathrm{H}) .{ }^{13} \mathrm{C}$ NMR (100 MHz, $\mathrm{CDCl}_{3}$ ) $\delta$ 177.2, 159.2, 157.4 (br), 144.3, 136.3 (2C), 131.6 (2C), 130.7, 130.3, 129.5 (3C), 128.3, 127.9 (2C), 123.5, 114.7 (2C), 110.0, 81.7, 68.3, 61.1, 56.0, 44.1 (2C), 40.4, 29.1 (3C). HRMS (ESI) $m / z: 522.2368[\mathrm{M}+\mathrm{Na}]^{+}$; calcd for $\mathrm{C}_{30} \mathrm{H}_{33} \mathrm{~N}_{3} \mathrm{NaO}_{4}{ }^{+}$, 522.2363. Diastereoisomer $6 \mathbf{b}^{\prime} .{ }^{1} \mathrm{H}$ NMR $\left(300 \mathrm{MHz}, \mathrm{CDCl}_{3}\right) \delta 7.41-$ 7.09 (m, 9H), 7.03-6.92 (m, 2H), 6.84 (d, br, $J=7.7 \mathrm{~Hz}, 1 \mathrm{H}), 6.70$ (d, br, $J=7.8 \mathrm{~Hz}, 1 \mathrm{H}), 4.97$ (d, $J=15.6 \mathrm{~Hz}, 1 \mathrm{H}), 4.77$ (d, $J=$ $15.6 \mathrm{~Hz}, 1 \mathrm{H}), 4.71-4.57$ (m, br, $2 \mathrm{H}), 3.77$ (s, 3H), 3.30 (dd, $J=13.7$ and $4.9 \mathrm{~Hz}, 1 \mathrm{H}), 2.84(\mathrm{dd}, J=13.7$ and $9.8 \mathrm{~Hz}, 1 \mathrm{H}), 2.35(\mathrm{dd}, J=$ 12.7 and $8.8 \mathrm{~Hz}, 1 \mathrm{H}), 2.24(\mathrm{dd}, J=12.7$ and $7.8 \mathrm{~Hz}, 1 \mathrm{H}), 1.51(\mathrm{~s}$, 9H). ${ }^{13} \mathrm{C}$ NMR (100 MHz, $\left.\mathrm{CDCl}_{3}\right) \delta 174.8,159.1,152.9$ (br), 142.5, 135.8, 132.3, 131.0 (2C), 130.5, 129.5 (3C), 128.4, 127.9 (2C), 124.0, 123.4, 114.7 (2C), 110.3, 81.6, 68.6, 61.8, 55.9, 45.1, 44.9, 40.8, 29.1 (3C). HRMS (ESI) $m / z: 522.2367[\mathrm{M}+\mathrm{Na}]^{+}$; calcd for $\mathrm{C}_{30} \mathrm{H}_{33} \mathrm{~N}_{3} \mathrm{NaO}_{4}{ }^{+}, 522.2363$.

$\left(3 S^{*}, 5^{\prime} S^{*}\right)$-tert-Butyl 1-benzyl-5'-(4-nitrobenzyl)-2-oxospiro [indoline-3, $3^{\prime}$-pyrazolidine]-1'-carboxylate $(6 \mathrm{c})$ and $\left(3 S^{*}, 5^{\prime} R^{*}\right)$ tert-butyl 1-benzyl-5' -(4-nitrobenzyl)-2-oxospiro[indoline-3,3'-py razolidine]-1'-carboxylate $\left(\mathbf{6 c}^{\prime}\right)$. Prepared according to TP-C starting from compound 5a and $p$-Br-nitrobenzene, and purified by FC (hexane : ethyl acetate $7: 3$ ). Yellow foam (53\% yield, separated diastereoisomers, $1.5: 1 \mathrm{dr})$.

Diastereoisomer 6c. ${ }^{1} \mathrm{H}$ NMR $\left(300 \mathrm{MHz}, \mathrm{CDCl}_{3}\right) \delta 8.18(\mathrm{~d}, \mathrm{br}, J$ $=8.8 \mathrm{~Hz}, 2 \mathrm{H}), 7.47(\mathrm{~d}, \mathrm{br}, J=8.8 \mathrm{~Hz}, 2 \mathrm{H}), 7.33-7.09(\mathrm{~m}, 7 \mathrm{H})$, 7.06-6.94 (m, 2H), 6.66 (d, br, $J=7.7 \mathrm{~Hz}, 1 \mathrm{H}), 4.93(\mathrm{~m}, \mathrm{br}, 1 \mathrm{H})$,
4.84 (d, br, $J=15.5 \mathrm{~Hz}, 1 \mathrm{H}), 4.76$ (d, br, $J=15.5 \mathrm{~Hz}, 1 \mathrm{H}), 3.35$ (dd, $J=13.2$ and $5.8 \mathrm{~Hz}, 1 \mathrm{H}), 3.09$ (dd, $J=13.2$ and $7.8 \mathrm{~Hz}, 1 \mathrm{H}$ ), $2.54(\mathrm{dd}, J=12.7$ and $7.8 \mathrm{~Hz}, 1 \mathrm{H}), 2.09(\mathrm{dd}, J=13.2$ and $8.8 \mathrm{~Hz}$, $1 \mathrm{H}), 1.47$ (s, 9H). ${ }^{13} \mathrm{C} \mathrm{NMR}\left(75 \mathrm{MHz}, \mathrm{CDCl}_{3}\right) \delta 176.3,156.9(\mathrm{br})$, 146.9, 145.9, 143.8, 135.6, 130.5 (2C), 130.2, 130.0, 128.8 (2C), 127.7, 127.2 (2C), 123.7 (2C), 122.8, 122.4, 109.6, 81.1, 66.4, 60.2, 43.4, 42.3, 41.2, 28.3 (3C). HRMS (ESI) $m / z: 537.2113[\mathrm{M}+\mathrm{Na}]^{+}$; calcd for $\mathrm{C}_{29} \mathrm{H}_{30} \mathrm{~N}_{4} \mathrm{NaO}_{5}{ }^{+}$, 537.2108. Diastereoisomer $6 \mathbf{c}^{\prime}$. ${ }^{1} \mathrm{H}$ $\operatorname{NMR}\left(400 \mathrm{MHz}, \mathrm{CDCl}_{3}\right) \delta 8.20(\mathrm{~d}, \mathrm{br}, J=8.8 \mathrm{~Hz}, 2 \mathrm{H}), 7.48(\mathrm{~d}, \mathrm{br}, J$ $=8.8 \mathrm{~Hz}, 2 \mathrm{H}), 7.38-7.15(\mathrm{~m}, 7 \mathrm{H}), 7.04-6.97(\mathrm{~m}, 2 \mathrm{H}), 6.76(\mathrm{~d}, \mathrm{br}, J$ $=7.7 \mathrm{~Hz}, 1 \mathrm{H}), 5.01(\mathrm{~d}, J=15.5 \mathrm{~Hz}, 1 \mathrm{H}), 4.80(\mathrm{~d}, J=15.5 \mathrm{~Hz}, 1 \mathrm{H})$, $4.74(\mathrm{~m}, \mathrm{br}, 1 \mathrm{H}), 3.47(\mathrm{dd}, J=13.2$ and $5.0 \mathrm{~Hz}, 1 \mathrm{H}), 3.06(\mathrm{dd}, J=$ 13.2 and $8.5 \mathrm{~Hz}, 1 \mathrm{H}), 2.41-2.30(\mathrm{~m}, 2 \mathrm{H}), 1.50(\mathrm{~s}, 9 \mathrm{H}) .{ }^{13} \mathrm{C} \mathrm{NMR}$ $\left(100 \mathrm{MHz}, \mathrm{CDCl}_{3}\right) \delta 174.7,153.1$ (br), 147.6, 146.3, 142.5, 135.7, 132.1, 130.9 (2C), 129.8, 129.6 (2C), 128.6, 128.0 (2C), 124.5 (2C), 124.1, 123.4, 110.5, 82.1, 68.8, 61.3, 45.2, 45.0, 41.6, 29.0 (3C). HRMS (ESI) $m / z: 537.2102[\mathrm{M}+\mathrm{Na}]^{+}$; calcd for $\mathrm{C}_{29} \mathrm{H}_{30} \mathrm{~N}_{4} \mathrm{NaO}_{5}{ }^{+}$, 537.2108 .

$\left(3 S^{*}, 5^{\prime} S^{*}\right)$-tert-Butyl $5^{\prime}$-(4-benzoylbenzyl)-1-benzyl-2-oxospiro [indoline-3, $3^{\prime}$-pyrazolidine]-1'-carboxylate (6d) and $\left(3 S^{*}, 5^{\prime} R^{*}\right)$ tert-butyl $5^{\prime}$-(4-benzoylbenzyl)-1-benzyl-2-oxospiro[indoline-3, $3^{\prime}$ -pyrazolidine]-1'-carboxylate $\left(\mathbf{6 d}^{\prime}\right)$. Prepared according to TP-C starting from compound $\mathbf{5 a}$ and $p$-Br-benzophenone, and purified by FC (hexane : ethyl acetate $7: 3)$. Yellow foam $(92 \%$ yield, separated diastereoisomers, $1: 1 \mathrm{dr}$ ). Diastereoisomer $6 \mathbf{d}$. ${ }^{1} \mathrm{H}$ NMR $\left(300 \mathrm{MHz}, \mathrm{CDCl}_{3}\right) \delta 7.80(\mathrm{~m}, 2 \mathrm{H}), 7.62-7.18(\mathrm{~m}, 12 \mathrm{H})$, $7.07(\mathrm{td}, J=7.8$ and $1.9 \mathrm{~Hz}, 1 \mathrm{H}), 6.95(\mathrm{t}, \mathrm{br}, J=7.8 \mathrm{~Hz}, 1 \mathrm{H}), 6.88$ (m, br, 1H), 6.65 (d, J= 7.8 Hz, 1H), $4.99(\mathrm{~m}, \mathrm{br}, 1 \mathrm{H}), 4.84$ (d, br, $=15.6 \mathrm{~Hz}, 1 \mathrm{H}), 4.76(\mathrm{~d}, \mathrm{br}, J=15.6 \mathrm{~Hz}, 1 \mathrm{H}), 4.49-4.15(\mathrm{~m}, \mathrm{br}$, $1 \mathrm{H}), 3.30(\mathrm{dd}, J=13.7$ and $4.9 \mathrm{~Hz}, 1 \mathrm{H}), 3.11$ (dd, $J=13.7$ and $7.8 \mathrm{~Hz}, 1 \mathrm{H}), 2.56(\mathrm{dd}, J=13.7$ and $8.8 \mathrm{~Hz}, 1 \mathrm{H}), 2.15(\mathrm{dd}, J=13.7$ and $8.8 \mathrm{~Hz}, 1 \mathrm{H}), 1.52(\mathrm{~s}, 9 \mathrm{H}) .{ }^{13} \mathrm{C} \mathrm{NMR}\left(100 \mathrm{MHz} \mathrm{CDCl}_{3}\right) \delta 197.0$, 177.1, 157.4 (br), 144.4, 144.2, 143.6, 138.4, 136.8, 136.3, 133.1, 131.1 (2C), 130.7, 130.6 (2C), 130.4 (2C), 129.5 (2C), 129.0 (2C), 128.3, 127.9 (2C), 123.4, 123.1, 110.1, 81.6, 67.2, 60.9, 44.1 (2C), 41.8, 29.1 (3C). HRMS (ESI) $m / z: 596.2526[\mathrm{M}+\mathrm{Na}]^{+}$; calcd for $\mathrm{C}_{36} \mathrm{H}_{35} \mathrm{~N}_{3} \mathrm{NaO}_{4}{ }^{+}$, 596.2520. Diastereoisomer $6 \mathbf{d}^{\prime} .{ }^{1} \mathrm{H}$ NMR (300 $\left.\mathrm{MHz} \mathrm{CDCl}_{3}\right) \delta 7.82-7.20(\mathrm{~m}, 15 \mathrm{H}), 7.15(\mathrm{td}, J=7.8$ and $1.9 \mathrm{~Hz}$, $1 \mathrm{H}), 6.97(\mathrm{t}, \mathrm{br}, J=7.8 \mathrm{~Hz}, 1 \mathrm{H}), 6.73(\mathrm{~d}, J=7.7 \mathrm{~Hz}, 1 \mathrm{H}), 4.99(\mathrm{~d}, J$ $=15.6 \mathrm{~Hz}, 1 \mathrm{H}), 4.78(\mathrm{~d}, J=15.6 \mathrm{~Hz}, 1 \mathrm{H}), 4.78-4.66(\mathrm{~m}, \mathrm{br}, 2 \mathrm{H})$, $3.46(\mathrm{dd}, J=12.7$ and $4.9 \mathrm{~Hz}, 1 \mathrm{H}), 2.99(\mathrm{dd}, J=12.7$ and $8.5 \mathrm{~Hz}$, $1 \mathrm{H}), 2.36(\mathrm{dd}, J=12.7$ and $7.8 \mathrm{~Hz}, 1 \mathrm{H}), 2.30(\mathrm{dd}, J=12.7$ and $7.8 \mathrm{~Hz}, 1 \mathrm{H}), 1.51(\mathrm{~s}, 9 \mathrm{H}) .{ }^{13} \mathrm{C} \mathrm{NMR}\left(100 \mathrm{MHz}, \mathrm{CDCl}_{3}\right) \delta 197.0$, 174.8, 156.7 (br), 143.5, 142.5, 138.4, 136.8, 135.8, 133.0, 132.2, 131.2 (2C), 130.6 (2C), 130.0 (2C), 129.7, 129.6 (2C), 128.9 (2C), 128.5, 127.9 (2C), 124.0, 123.4, 110.4, 81.9, 68.7, 61.5, 45.3, 45.0, 41.9, 29.1 (3C). HRMS (ESI) $m / z: 596.2527[\mathrm{M}+\mathrm{Na}]^{+}$; calcd for $\mathrm{C}_{36} \mathrm{H}_{35} \mathrm{~N}_{3} \mathrm{NaO}_{4}{ }^{+}, 596.2520$.

$\left(3 S^{*}, 5^{\prime} S^{*}\right)$-tert-Butyl $\quad 5^{\prime}$-(4-acetylbenzyl)-1-benzyl-2-oxospiro [indoline-3, $3^{\prime}$-pyrazolidine]-1'-carboxylate (6e) and $\left(3 S^{*}, 5^{\prime} R^{*}\right)$ tert-butyl $5^{\prime}$-(4-acetylbenzyl)-1-benzyl-2-oxospiro[indoline-3, $3^{\prime}$ pyrazolidine]-1'-carboxylate $\left(6 \mathbf{e}^{\prime}\right)$. Prepared according to TP-C starting from compound 5a and $p$-Br-acetophenone, and purified by FC (hexane : ethyl acetate $7: 3$ ). Yellow foam (98\% yield, separated diastereoisomers, $1: 1 \mathrm{dr}$ ). Diastereoisomer 6 e. ${ }^{1} \mathrm{H}$ NMR (300 MHz, $\left.\mathrm{CDCl}_{3}\right) \delta 7.93(\mathrm{~d}, J=8.8 \mathrm{~Hz}, 2 \mathrm{H}), 7.39(\mathrm{~d}, J=$ $8.8 \mathrm{~Hz}, 2 \mathrm{H}), 7.33-7.21(\mathrm{~m}, 5 \mathrm{H}), 7.15(\mathrm{t}, J=7.8 \mathrm{~Hz}, 1 \mathrm{H}), 6.94(\mathrm{t}, J=$ 
$7.8 \mathrm{~Hz}, 1 \mathrm{H}), 6.85$ (d, br, $J=7.8 \mathrm{~Hz}, 1 \mathrm{H}), 6.64(\mathrm{~d}, J=7.8 \mathrm{~Hz}, 1 \mathrm{H})$, $4.95(\mathrm{~m}, \mathrm{br}, 1 \mathrm{H}), 4.83$ (d, br, $J=15.6 \mathrm{~Hz}, 1 \mathrm{H}), 4.75$ (d, br, $J=$ $15.6 \mathrm{~Hz}, 1 \mathrm{H}), 4.27$ (m, br, $1 \mathrm{H}), 3.26$ (dd, $J=13.7$ and $4.9 \mathrm{~Hz}, 1 \mathrm{H}$ ), $3.07(\mathrm{dd}, J=13.7$ and $7.8 \mathrm{~Hz}, 1 \mathrm{H}), 2.60(\mathrm{~s}, 3 \mathrm{H}), 2.52(\mathrm{dd}, J=12.7$ and $7.8 \mathrm{~Hz}, 1 \mathrm{H}), 2.10(\mathrm{dd}, J=12.7$ and $8.8 \mathrm{~Hz}, 1 \mathrm{H}), 1.50(\mathrm{~s}, 9 \mathrm{H})$. ${ }^{13} \mathrm{C}$ NMR (100 MHz, $\mathrm{CDCl}_{3}$ ) $\delta$ 197.9, 176.3, 156.8 (br), $143.6(2 \mathrm{C})$, 135.7, 135.6, 130.1 (3C), 128.8 (2C), 128.6 (2C), 127.6, 127.2 (2C), 125.2, 122.8, 122.5, 109.4, 80.9, 66.4, 60.2, 43.4, 41.9 (br), 41.0, 28.4 (3C), 26.7. HRMS (ESI) $m / z: 534.2355[\mathrm{M}+\mathrm{Na}]^{+}$; calcd for $\mathrm{C}_{31} \mathrm{H}_{33} \mathrm{~N}_{3} \mathrm{NaO}_{4}{ }^{+}$, 534.2363. Diastereoisomer $6 \mathrm{e}^{\prime} .{ }^{1} \mathrm{H}$ NMR $(300$ $\left.\mathrm{MHz}, \mathrm{CDCl}_{3}\right) \delta 7.89(\mathrm{~d}, J=8.8 \mathrm{~Hz}, 2 \mathrm{H}), 7.44-7.20(\mathrm{~m}, 7 \mathrm{H}), 7.14(\mathrm{t}$, $J=7.8 \mathrm{~Hz}, 1 \mathrm{H}), 7.01-6.92(\mathrm{~m}, 2 \mathrm{H}), 6.71(\mathrm{~d}, J=7.8 \mathrm{~Hz}, 1 \mathrm{H}), 4.97$ $(\mathrm{d}, J=15.6 \mathrm{~Hz}, 1 \mathrm{H}), 4.76(\mathrm{~d}, J=15.6 \mathrm{~Hz}, 1 \mathrm{H}), 4.70(\mathrm{~m}, \mathrm{br}, 1 \mathrm{H})$, 4.65 (s, br, 1H), 3.42 (dd, $J=13.7$ and $4.9 \mathrm{~Hz}, 1 \mathrm{H}), 2.95$ (dd, $J=$ 13.7 and $8.8 \mathrm{~Hz}, 1 \mathrm{H}), 2.57(\mathrm{~s}, 3 \mathrm{H}), 2.32(\mathrm{dd}, J=12.7$ and $8.8 \mathrm{~Hz}$, $1 \mathrm{H}), 2.26(\mathrm{dd}, J=12.7$ and $7.8 \mathrm{~Hz}, 1 \mathrm{H}), 1.49(\mathrm{~s}, 9 \mathrm{H}) .{ }^{13} \mathrm{C} \mathrm{NMR}$ $\left(100 \mathrm{MHz} \mathrm{CDCl}_{3}\right) \delta 197.8,174.1,156.2$ (br), 143.5, 141.8, 135.7, 135.1, 131.4, 129.6 (2C), 129.0, 128.9, 128.7 (2C), 127.9 (2C), 127.3 (2C), 123.4, 122.7, 109.8, 81.2, 68.0, 60.7, 44.5, 44.2, 41.0, 28.3 (3C), 26.6. HRMS (ESI) $m / z: 5342.358[\mathrm{M}+\mathrm{Na}]^{+}$; calcd for $\mathrm{C}_{31} \mathrm{H}_{33} \mathrm{~N}_{3} \mathrm{NaO}_{4}{ }^{+}, 534.2363$.

$\left(3 S^{*}, 5^{\prime} S^{*}\right)$-tert-Butyl 1-benzyl-5'-(2-methylbenzyl)-2-oxospiro [indoline-3, $3^{\prime}$-pyrazolidine]-1'-carboxylate (6f) and $\left(3 S^{*}, 5^{\prime} R^{*}\right)$ tert-butyl 1-benzyl-5' -(2-methylbenzyl)-2-oxospiro[indoline-3,3' pyrazolidine]-1'-carboxylate $\left(\mathbf{6 f}^{\prime}\right)$. Prepared according to TP-C starting from compound 5a and o-Br-toluene, and purified by FC (hexane : ethyl acetate $7: 3$ ). Yellow foam (98\% yield, separated diastereoisomers, 1 : $1.6 \mathrm{dr}$ ). Diastereoisomer $6 \mathbf{6 f}{ }^{1} \mathrm{H}$ NMR $\left(300 \mathrm{MHz}, \mathrm{CDCl}_{3}\right) \delta 7.35-7.10(\mathrm{~m}, 10 \mathrm{H}), 7.01-6.89(\mathrm{~m}, 2 \mathrm{H}), 6.63$ $(\mathrm{d}, J=7.8 \mathrm{~Hz}, 1 \mathrm{H}), 4.95(\mathrm{~m}, 1 \mathrm{H}), 4.88(\mathrm{~d}, \mathrm{br}, J=15.6 \mathrm{~Hz}, 1 \mathrm{H})$, 4.73 (d, br, $J=15.6 \mathrm{~Hz}, 1 \mathrm{H}), 4.41$ (m, br, $1 \mathrm{H}), 3.28$ (dd, $J=13.7$ and $4.9 \mathrm{~Hz}, 1 \mathrm{H}), 2.98(\mathrm{dd}, J=13.7$ and $7.8 \mathrm{~Hz}, 1 \mathrm{H}), 2.50(\mathrm{dd}, J=$ 12.7 and $7.8 \mathrm{~Hz}, 1 \mathrm{H}), 2.42(\mathrm{~s}, 3 \mathrm{H}), 2.13(\mathrm{dd}, J=12.7$ and $7.8 \mathrm{~Hz}$, 1H), 1.49 (s, 9H). ${ }^{13} \mathrm{C}$ NMR (100 MHz, $\left.\mathrm{CDCl}_{3}\right) \delta 176.6,156.7$ (br), 143.7, 137.0, 136.3, 135.7, 130.5 (2C), 129.9, 128.8 (2C), 127.6, 127.2 (2C), 126.8, 126.0, 125.7, 122.7, 122.6, 109.4, 80.7, 66.5, 59.3, 43.3, 42.0, 38.5, 28.4 (3C), 19.9. HRMS (ESI) $m / z: 506.2411$ $[\mathrm{M}+\mathrm{Na}]^{+}$; calcd for $\mathrm{C}_{30} \mathrm{H}_{33} \mathrm{~N}_{3} \mathrm{NaO}_{3}{ }^{+}, 506.2414$. Diastereoisomer 6f' ${ }^{1} \mathrm{H}$ NMR (300 MHz, $\left.\mathrm{CDCl}_{3}\right) \delta$ 7.35-7.08 (m, 10H), 7.03-6.92 $(\mathrm{m}, 2 \mathrm{H}), 6.70(\mathrm{~d}, J=7.8 \mathrm{~Hz}, 1 \mathrm{H}), 4.98(\mathrm{~d}, J=15.6 \mathrm{~Hz}, 1 \mathrm{H}), 4.84-$ $4.67(\mathrm{~m}, 3 \mathrm{H}), 3.41(\mathrm{dd}, J=12.7$ and $4.9 \mathrm{~Hz}, 1 \mathrm{H}), 2.87(\mathrm{dd}, J=12.7$ and $8.8 \mathrm{~Hz}, 1 \mathrm{H}), 2.45(\mathrm{~s}, 3 \mathrm{H}), 2.37(\mathrm{dd}, J=12.7$ and $7.8 \mathrm{~Hz}, 1 \mathrm{H})$, $2.25(\mathrm{dd}, J=12.7$ and $7.8 \mathrm{~Hz}, 1 \mathrm{H}), 1.47$ (s, 9H). ${ }^{13} \mathrm{C}$ NMR (100 $\left.\mathrm{MHz}, \mathrm{CDCl}_{3}\right) \delta 174.3,156.1$ (br), 141.8, 136.6, 136.2, 135.1, 131.7, 130.4 (2C), 128.9 (3C), 127.8, 127.3 (2C), 126.8, 126.1, 123.3, 122.7, 109.7, 81.1, 68.2, 59.6, 44.7, 44.2, 38.8, 28.4 (3C), 19.8. HRMS (ESI) $m / z: 506.2419[\mathrm{M}+\mathrm{Na}]^{+}$; calcd for $\mathrm{C}_{30} \mathrm{H}_{33} \mathrm{~N}_{3} \mathrm{NaO}_{3}{ }^{+}, 506.2414$.

$\left(3 S^{*}, 5^{\prime} S^{*}\right)$-tert-Butyl 1-benzyl-2-oxo-5'-(2,4,6-trimethylbenzyl) spiro[indoline-3, $3^{\prime}$-pyrazolidine]-1'-carboxylate $(6 \mathrm{~g})$ and $\left(3 S^{*}, 5^{\prime} R^{*}\right)$ -tert-butyl 1-benzyl-2-oxo-5'-(2,4,6-trimethylbenzyl)spiro[indoline$3,3^{\prime}$-pyrazolidine]-1'-carboxylate $\left(6 \mathbf{g}^{\prime}\right)$. Prepared according to TP-C starting from compound 5a and 2-Br-1,3,5-trimethylbenzene, and purified by FC (hexane : ethyl acetate $7: 3$ ). Yellow foam (70\% yield, separated diastereoisomers, $1: 1 \mathrm{dr}$ ). Diastereoisomer 6g. ${ }^{1} \mathrm{H}$ NMR $\left(300 \mathrm{MHz}, \mathrm{CDCl}_{3}\right) \delta 7.32-7.20(\mathrm{~m}, 6 \mathrm{H}), 7.09-$ $6.98(\mathrm{~m}, 2 \mathrm{H}), 6.84(\mathrm{~s}, 2 \mathrm{H}), 6.65(\mathrm{~d}, J=7.8 \mathrm{~Hz}, 1 \mathrm{H}), 5.01-4.86(\mathrm{~m}$,
$3 \mathrm{H}), 4.69(\mathrm{~d}, J=15.6 \mathrm{~Hz}, 1 \mathrm{H}), 3.28(\mathrm{dd}, J=13.7$ and $7.8 \mathrm{~Hz}, 1 \mathrm{H})$, $3.04(\mathrm{dd}, J=13.7$ and $6.8 \mathrm{~Hz}, 1 \mathrm{H}), 3.28(\mathrm{dd}, J=12.7$ and $7.8 \mathrm{~Hz}$, $1 \mathrm{H}), 2.40(\mathrm{~s}, 6 \mathrm{H}), 2.38-2.29(\mathrm{~m}, 1 \mathrm{H}), 2.24(\mathrm{~s}, 3 \mathrm{H}), 1.37(\mathrm{~s}, 9 \mathrm{H}) .{ }^{13} \mathrm{C}$ NMR $\left(100 \mathrm{MHz}, \mathrm{CDCl}_{3}\right) \delta 177.4,157.0,144.4,137.5,136.4,136.2$, 133.0, 130.6, 129.8 (3C), 129.6 (2C), 129.1, 128.3, 127.9, 126.6, 123.5, 123.4, 110.1, 81.2, 67.2, 59.4, 44.4, 44.1, 35.7, 28.8 (3C), 21.5, 21.4 (2C). HRMS (ESI) $m / z: 534.2721[\mathrm{M}+\mathrm{Na}]^{+}$; calcd for $\mathrm{C}_{32} \mathrm{H}_{37} \mathrm{~N}_{3} \mathrm{NaO}_{3}{ }^{+}$, 534.2727. Diastereoisomer $6 \mathbf{g}^{\prime}$. 1H NMR (300 $\left.\mathrm{MHz} \mathrm{CDCl}_{3}\right) \delta 7.35-7.21(\mathrm{~m}, 5 \mathrm{H}), 7.14(\mathrm{td}, J=7.8$ and $2.9 \mathrm{~Hz}$, 1H), 7.02-6.92 (m, 2H), $6.83(\mathrm{~s}, 2 \mathrm{H}), 6.70$ (d, $J=7.8 \mathrm{~Hz}, 1 \mathrm{H}), 4.96$ $(\mathrm{d}, J=15.6 \mathrm{~Hz}, 1 \mathrm{H}), 4.93-4.76(\mathrm{~m}, 3 \mathrm{H}), 3.29(\mathrm{dd}, J=13.7$ and $6.8 \mathrm{~Hz}, 1 \mathrm{H}), 2.96(\mathrm{dd}, J=13.7$ and $6.8 \mathrm{~Hz}, 1 \mathrm{H}), 2.41(\mathrm{~s}, 6 \mathrm{H}), 2.38-$ $2.29(\mathrm{~m}, 2 \mathrm{H}), 2.23(\mathrm{~s}, 3 \mathrm{H}), 1.36(\mathrm{~s}, 9 \mathrm{H}) .{ }^{13} \mathrm{C} \mathrm{NMR}(100 \mathrm{MHz}$, $\left.\mathrm{CDCl}_{3}\right) \delta 175.1,156.5$ (br), 142.5, 137.5 (2C), 136.3, 135.8, 132.8, 132.6, 129.8 (3C), 129.6 (3C), 128.5, 128.0, 124.0, 123.4, 110.4, 81.5, 69.0, 59.7, 45.3, 44.9, 35.4, 28.8 (3C), 21.5, 21.4 (2C). HRMS (ESI) $m / z: 534.2719[\mathrm{M}+\mathrm{Na}]^{+}$; calcd for $\mathrm{C}_{32} \mathrm{H}_{37} \mathrm{~N}_{3} \mathrm{NaO}_{3}{ }^{+}$, 534.2727 .

$\left(3 S^{*}, 5^{\prime} S^{*}\right)$-tert-Butyl 1-benzyl-5' -(4-methylbenzyl)-2-oxospiro [indoline-3, $3^{\prime}$-pyrazolidine]-1'-carboxylate $(6 \mathrm{~h})$ and $\left(3 S^{*}, 5^{\prime} R^{*}\right)$ tert-butyl 1-benzyl-5' -(4-methylbenzyl)-2-oxospiro[indoline-3, $3^{\prime}$ pyrazolidine]-1'-carboxylate $\left(\mathbf{6 h}^{\prime}\right)$. Prepared according to TP-C starting from compound 5a and $p$-I-toluene, and purified by FC (hexane : ethyl acetate $7: 3$ ). Yellow foam (63\% yield, separated diastereoisomers, 1 : $1 \mathrm{dr}$ ). Diastereoisomer $6 \mathbf{h} .{ }^{1} \mathrm{H}$ NMR $\left(300 \mathrm{MHz}, \mathrm{CDCl}_{3}\right) \delta 7.32-7.10(\mathrm{~m}, 10 \mathrm{H}), 6.92(\mathrm{t}, J=7.8 \mathrm{~Hz}, 1 \mathrm{H})$, $6.77(\mathrm{~m}, \mathrm{br}, 1 \mathrm{H}), 6.61(\mathrm{~d}, J=7.8 \mathrm{~Hz}, 1 \mathrm{H}), 4.98-4.67(\mathrm{~m}, \mathrm{br}, 4 \mathrm{H})$, $3.12(\mathrm{dd}, J=13.6$ and $3.9 \mathrm{~Hz}, 1 \mathrm{H}), 3.03(\mathrm{dd}, J=13.6$ and $6.8 \mathrm{~Hz}$, $1 \mathrm{H}), 2.51(\mathrm{dd}, J=12.7$ and $7.8 \mathrm{~Hz}, 1 \mathrm{H}), 2.34(\mathrm{~s}, 3 \mathrm{H}), 2.17$ (dd, $J=$ 12.7 and $7.8 \mathrm{~Hz}, 1 \mathrm{H}), 1.52(\mathrm{~s}, 9 \mathrm{H}) .{ }^{13} \mathrm{C} \mathrm{NMR}\left(100 \mathrm{MHz}, \mathrm{CDCl}_{3}\right.$ ) $\delta 177.1,157.3,144.3,136.9,136.3,135.2,130.6,130.5$ (2C), 130.0 (2C), 129.5 (2C), 128.3, 127.9 (2C), 122.3 (2C), 121.3, 110.0, 81.5, 67.3, 61.0, 44.5, 44.0, 41.0, 29.1 (3C), 21.7. HRMS (ESI) $m / z$ : 506.2410 $[\mathrm{M}+\mathrm{Na}]^{+}$; calcd for $\mathrm{C}_{30} \mathrm{H}_{33} \mathrm{~N}_{3} \mathrm{NaO}_{3}{ }^{+}$, 506.2414. Diastereoisomer 6h'. ${ }^{1} \mathrm{H}$ NMR (400 $\left.\mathrm{MHz}, \mathrm{CDCl}_{3}\right) \delta 7.36-7.10(\mathrm{~m}$, $10 \mathrm{H}$ ), 7.05-6.95 (m, 2H), 6.73 (d, $J=7.8 \mathrm{~Hz}, 1 \mathrm{H}), 5.00$ (d, $J=$ $15.6 \mathrm{~Hz}, 1 \mathrm{H}), 4.81$ (d, $J=15.6 \mathrm{~Hz}, 1 \mathrm{H}), 4.73-4.62$ (m, br, 2H), $3.37(\mathrm{dd}, J=12.7$ and $3.9 \mathrm{~Hz}, 1 \mathrm{H}), 2.88(\mathrm{dd}, J=12.7$ and $8.8 \mathrm{~Hz}$, $1 \mathrm{H}), 2.39(\mathrm{dd}, J=12.7$ and $8.8 \mathrm{~Hz}, 1 \mathrm{H}), 2.34(\mathrm{~s}, 3 \mathrm{H}), 2.28(\mathrm{dd}, J=$ 12.7 and $7.8 \mathrm{~Hz}, 1 \mathrm{H}), 1.55$ (s, 9H). ${ }^{13} \mathrm{C} \mathrm{NMR}\left(100 \mathrm{MHz}, \mathrm{CDCl}_{3}\right.$ ) $\delta 174.8,156.5,142.5,136.9,135.8,135.3,132.3,130.0$ (4C), 129.5 (3C), 128.5, 127.9 (2C), 124.0, 123.4, 110.3, 81.7, 68.6, 61.8, 45.2, 44.9, 41.3, 29.1 (3C), 21.7. HRMS (ESI) $m / z: 506.2409[\mathrm{M}+\mathrm{Na}]^{+}$; calcd for $\mathrm{C}_{30} \mathrm{H}_{33} \mathrm{~N}_{3} \mathrm{NaO}_{3}{ }^{+}, 506.2414$.

$\left(3 S^{*}, 5^{\prime} S^{*}\right)$-tert-Butyl $5^{\prime}$-([1,1'-biphenyl]-4-ylmethyl)-1-methyl-2oxospiro[indoline- $3,3^{\prime}$-pyrazolidine]-1'-carboxylate $(6 \mathrm{i})$ and $\left(3 S^{*}\right.$ , $\left.5^{\prime} R^{*}\right)$-tert-butyl 5'-([1,1'-biphenyl]-4-ylmethyl)-1-methyl-2-oxospiro [indoline-3,3' -pyrazolidine]-1' $\mathbf{1}^{\prime}$-carboxylate (6i'). Prepared according to TP-C starting from compound $\mathbf{5 b}$ and $p$-Br-biphenyl, and purified by FC (hexane : ethyl acetate $7: 3)$. Yellow foam $(53 \%$ yield, separated diastereoisomers, $1.5: 1 \mathrm{dr})$. Diastereoisomer 6i. ${ }^{1} \mathrm{H}$ NMR $\left(300 \mathrm{MHz}, \mathrm{CDCl}_{3}\right) \delta$ 7.62-7.52 (m, 4H), 7.49-7.22 (m, $7 \mathrm{H}), 6.96(\mathrm{t}, \mathrm{br}, J=7.8 \mathrm{~Hz}, 1 \mathrm{H}), 6.88(\mathrm{~m}, \mathrm{br}, 1 \mathrm{H}), 6.74(\mathrm{~d}, \mathrm{br}, J=$ $7.8 \mathrm{~Hz}, 1 \mathrm{H}), 4.88(\mathrm{~m}, \mathrm{br}, 1 \mathrm{H}), 3.24-3.12(\mathrm{~m}, 2 \mathrm{H}), 3.10(\mathrm{~s}, 3 \mathrm{H}), 2.49$ (dd, $J=12.7$ and $6.8 \mathrm{~Hz}, 1 \mathrm{H}), 2.17(\mathrm{dd}, J=12.7$ and $7.8 \mathrm{~Hz}, 1 \mathrm{H}$ ), $1.53(\mathrm{~s}, 9 \mathrm{H}) .{ }^{13} \mathrm{C} \mathrm{NMR}\left(100 \mathrm{MHz}, \mathrm{CDCl}_{3}\right) \delta 176.4,156.7$ (br), 144.6, 140.9, 139.7, 136.9, 130.3 (2C), 130.0, 128.8 (2C), 127.3 
(3C), 127.0 (2C), 125.6, 122.7, 122.4, 108.3, 80.8, 66.4, 60.2, 41.9, 40.5, 28.5 (3C), 26.0. HRMS (ESI) $m / z: 492.2262[\mathrm{M}+\mathrm{Na}]^{+}$; calcd for $\mathrm{C}_{29} \mathrm{H}_{31} \mathrm{~N}_{3} \mathrm{NaO}_{3}{ }^{+}, 492.2258$. Diastereoisomer $6 \mathrm{i}^{\prime} .{ }^{1} \mathrm{H}$ NMR $(300$ $\mathrm{MHz} \mathrm{CDCl}_{3}$ ) $\delta$ 7.58-7.47 (m, 4H), 7.46-7.28 (m, 5H), 7.09 (t, br, $=7.8 \mathrm{~Hz}, 1 \mathrm{H}), 6.99(\mathrm{~d}, \mathrm{br}, J=6.8 \mathrm{~Hz}, 2 \mathrm{H}), 6.83(\mathrm{~d}, \mathrm{br}, J=7.8 \mathrm{~Hz}$, $2 \mathrm{H}), 4.70(\mathrm{~m}, \mathrm{br}, 1 \mathrm{H}), 3.40(\mathrm{dd}, J=12.7$ and $4.9 \mathrm{~Hz}, 1 \mathrm{H}), 3.17(\mathrm{~s}$, $3 \mathrm{H}), 2.89(\mathrm{dd}, J=12.7$ and $9.8 \mathrm{~Hz}, 1 \mathrm{H}), 2.33(\mathrm{dd}, J=12.7$ and $8.8 \mathrm{~Hz}, 1 \mathrm{H}$ ), 2.24 (dd, $J=12.7$ and $7.8 \mathrm{~Hz}, 1 \mathrm{H}), 1.49(\mathrm{~s}, 9 \mathrm{H}) .{ }^{13} \mathrm{C}$ NMR (100 MHz, $\mathrm{CDCl}_{3}$ ) $\delta$ 173.9, 153.8 (br), 145.8, 140.9, 139.6, 137.0, 131.5, 130.7, 129.8 (2C), 128.8 (2C), 127.3 (2C), 127.2, 127.0 (2C), 123.3, 123.0, 108.7, 81.0, 68.1, 61.1, 44.4, 40.9, 28.4 (3C), 26.1. HRMS (ESI) $m / z: 492.2253[\mathrm{M}+\mathrm{Na}]^{+}$; calcd for $\mathrm{C}_{29} \mathrm{H}_{31} \mathrm{~N}_{3} \mathrm{NaO}_{3}{ }^{+}, 492.2258$.

$\left(3 S^{*}, 5^{\prime} S^{*}\right)$-tert-Butyl $5^{\prime}$-([1,1'-biphenyl $]-4$-ylmethyl)-1-((E)-3-(3, 4-dichlorophenyl)allyl)-2-oxospiro[indoline-3,3' -pyrazolidine]-1' carboxylate $(6 \mathrm{j})$ and $\left(3 S^{*}, 5^{\prime} R^{*}\right)$-tert-butyl $5^{\prime}$-([1,1'-biphenyl $]-4$ ylmethyl)-1-((E)-3-(3,4-dichlorophenyl)allyl)-2-oxospiro[indoline$\mathbf{3}, \mathbf{3}^{\prime}$-pyrazolidine]-1' $\mathbf{1}^{\prime}$-carboxylate $\left(\mathbf{6 j} \mathbf{j}^{\prime}\right)$. Prepared according to TP-C starting from compound $\mathbf{5 c}$ and $p$-Br-biphenyl, and purified by FC (hexane : ethyl acetate $7: 3$ ). Yellow foam (69\% yield, separated diastereoisomers, 1 : $1 \mathrm{dr}$ ). Diastereoisomer $6 \mathbf{6 j} .{ }^{1} \mathrm{H}$ NMR $\left(300 \mathrm{MHz}, \mathrm{CDCl}_{3}\right) \delta 7.62-7.54(\mathrm{~m}, 4 \mathrm{H}), 7.48-7.28(\mathrm{~m}, 8 \mathrm{H}), 7.23(\mathrm{t}$, $\mathrm{br}, J=7.8 \mathrm{~Hz}, 1 \mathrm{H}), 7.12(\mathrm{dd}, J=8.7$ and $1.9 \mathrm{~Hz}, 1 \mathrm{H}), 6.95(\mathrm{t}, \mathrm{br}, J$ $=7.8 \mathrm{~Hz}, 1 \mathrm{H}), 6.84-6.83(\mathrm{~m}, 2 \mathrm{H}), 6.44(\mathrm{~d}, \mathrm{br}, J=15.6 \mathrm{~Hz}, 1 \mathrm{H})$, $6.14(\mathrm{dt}, J=15.6$ and $4.9 \mathrm{~Hz}, 1 \mathrm{H}), 4.93(\mathrm{~m}, \mathrm{br}, 1 \mathrm{H}), 4.45$ (dd, br, $J$ $=16.6$ and $4.9 \mathrm{~Hz}, 1 \mathrm{H}), 4.31$ (dd, br, $J=16.6$ and $4.9 \mathrm{~Hz}, 1 \mathrm{H}$ ), $3.19(\mathrm{dd}, J=13.7$ and $3.9 \mathrm{~Hz}, 1 \mathrm{H}), 3.10(\mathrm{dd}, J=13.7$ and $6.8 \mathrm{~Hz}$, $1 \mathrm{H}), 2.54(\mathrm{dd}, J=12.7$ and $7.8 \mathrm{~Hz}, 1 \mathrm{H}), 2.17(\mathrm{dd}, J=12.7$ and $7.8 \mathrm{~Hz}, 1 \mathrm{H}), 1.52(\mathrm{~s}, 9 \mathrm{H}) .{ }^{13} \mathrm{C} \mathrm{NMR}\left(100 \mathrm{MHz}, \mathrm{CDCl}_{3}\right) \delta 176.2$, 154.4 (br), 143.6, 140.9, 139.8, 136.9, 136.3, 132.7, 131.6, 130.5 (2C), 130.4 (2C), 130.0, 128.9 (2C), 128.2, 127.3 (4C), 127.0, 125.7, 125.6, 125.0, 122.8, 122.6, 109.0, 80.8, 66.4, 60.3, 41.4 (2C), 40.5, 28.5 (3C). HRMS (ESI) $m / z: 662.1956[\mathrm{M}+\mathrm{Na}]^{+}$; calcd for $\mathrm{C}_{37} \mathrm{H}_{35} \mathrm{Cl}_{2} \mathrm{~N}_{3} \mathrm{NaO}_{3}{ }^{+}$, 662.1948. Diastereoisomer $6 \mathbf{j}^{\prime} \cdot{ }^{1} \mathrm{H}$ NMR $\left(300 \mathrm{MHz}, \mathrm{CDCl}_{3}\right) \delta 7.61-7.20(\mathrm{~m}, 13 \mathrm{H}), 7.15(\mathrm{~d}, \mathrm{br}, J=8.7 \mathrm{~Hz}$, $1 \mathrm{H}), 7.06-6.97(\mathrm{~m}, 2 \mathrm{H}), 6.85(\mathrm{~d}, J=7.8 \mathrm{~Hz}, 1 \mathrm{H}), 6.48$ (d, br, $J=$ $15.6 \mathrm{~Hz}, 1 \mathrm{H}), 6.15(\mathrm{dt}, J=15.6$ and $5.9 \mathrm{~Hz}, 1 \mathrm{H}), 4.71(\mathrm{~m}, \mathrm{br}, 1 \mathrm{H})$, 4.57-4.37 (m, 2H), $3.42(\mathrm{dd}, J=12.7$ and $3.9 \mathrm{~Hz}, 1 \mathrm{H}), 2.90$ (dd, $J$ $=12.7$ and $8.8 \mathrm{~Hz}, 1 \mathrm{H}), 2.43-2.24(\mathrm{~m}, 2 \mathrm{H}), 1.51(\mathrm{~s}, 9 \mathrm{H}) .{ }^{13} \mathrm{C} \mathrm{NMR}$ $\left(100 \mathrm{MHz}, \mathrm{CDCl}_{3}\right) \delta 173.8,151.0(\mathrm{br}), 141.7,140.9,139.6,136.9$, 136.1, 132.8, 131.7, 131.6, 131.0, 130.5, 129.8 (2C), 129.1, 128.8 (2C), 128.3, 127.3 (2C), 127.2, 127.0 (2C), 125.6, 124.5, 123.5, 122.9, 109.3, 81.1, 68.0, 61.2, 44.6, 42.2, 40.9, 28.4 (3C). HRMS (ESI) $m / z: 662.1954[\mathrm{M}+\mathrm{Na}]^{+}$; calcd for $\mathrm{C}_{37} \mathrm{H}_{35} \mathrm{Cl}_{2} \mathrm{~N}_{3} \mathrm{NaO}_{3}{ }^{+}$, 662.1948 .

$\left(3 S^{*}, 5^{\prime} S^{*}\right)$-tert-Butyl $5^{\prime}$-([1,1'-biphenyl]-4-ylmethyl)-1-benzyl-5methyl-2-oxospiro[indoline-3,3'-pyrazolidine]-1'-carboxylate (6k) and $\left(3 S^{*}, 5^{\prime} R^{*}\right)$-tert-butyl $5^{\prime}$-([1,1'-biphenyl $]-4$-ylmethyl)-1-benzyl -5-methyl-2-oxospiro[indoline-3, $3^{\prime}$-pyrazolidine]-1'-carboxylate $\left(\mathbf{6} \mathbf{k}^{\prime}\right)$. Prepared according to TP-C starting from compound $\mathbf{5 d}$ and $p$-Br-biphenyl, and purified by FC (hexane : ethyl acetate 7 : 3). Yellow foam (50\% yield, separated diastereoisomers, $1: 1$ dr). Diastereoisomer 6k. ${ }^{1} \mathrm{H}$ NMR $\left(300 \mathrm{MHz}, \mathrm{CDCl}_{3}\right) \delta 7.58$ (d, br, $J=7.8 \mathrm{~Hz}, 4 \mathrm{H}), 7.49-7.20(\mathrm{~m}, 12 \mathrm{H}), 6.90$ (d, br, $J=7.8 \mathrm{~Hz}, 1 \mathrm{H})$, 6.49 (d, $J=7.8 \mathrm{~Hz}, 1 \mathrm{H}), 5.02$ (m, br, 1H), 4.99-4.71 (m, br, 2H), $3.19(\mathrm{dd}, J=12.7$ and $3.9 \mathrm{~Hz}, 1 \mathrm{H}), 3.11(\mathrm{dd}, J=12.7$ and $6.8 \mathrm{~Hz}$, $1 \mathrm{H}), 2.56(\mathrm{dd}, J=12.7$ and $7.8 \mathrm{~Hz}, 1 \mathrm{H}), 2.19(\mathrm{dd}, J=12.7$ and
$7.8 \mathrm{~Hz}, 1 \mathrm{H}), 2.14$ (s, 3H), 1.53 (s, 9H). ${ }^{13} \mathrm{C} \mathrm{NMR}\left(100 \mathrm{MHz}, \mathrm{CDCl}_{3}\right)$ $\delta 177.1,158.5,141.2,140.8,139.8,137.0,135.8,132.3,130.5$ (2C), 130.1, 128.8 (2C), 128.7 (2C), 127.5, 127.3 (5C), 127.2, 127.0, 125.7, 123.3, 109.1, 80.7, 66.7, 60.3, 43.4 (2C), 40.6, 28.4 (3C), 20.9. HRMS (ESI) $m / z: 582.2723[\mathrm{M}+\mathrm{Na}]^{+}$; calcd for $\mathrm{C}_{36} \mathrm{H}_{37} \mathrm{~N}_{3} \mathrm{NaO}_{3}{ }^{+}$, 582.2727. Diastereoisomer $6 \mathbf{k}^{\prime} .{ }^{1} \mathrm{H}$ NMR (400 $\left.\mathrm{MHz} \mathrm{CDCl}_{3}\right) \delta 7.65-7.54(\mathrm{~m}, 4 \mathrm{H}), 7.45(\mathrm{t}, \mathrm{br}, J=7.8 \mathrm{~Hz}, 2 \mathrm{H})$, $7.42-7.23(\mathrm{~m}, 9 \mathrm{H}), 6.97$ (d, br, $J=7.8 \mathrm{~Hz}, 1 \mathrm{H}), 6.85(\mathrm{~s}, \mathrm{br}, 1 \mathrm{H})$, $6.62(\mathrm{~d}, J=7.8 \mathrm{~Hz}, 1 \mathrm{H}), 4,98(\mathrm{~d}, J=15.8 \mathrm{~Hz}, 1 \mathrm{H}), 4,80(\mathrm{~d}, J=$ $15.8 \mathrm{~Hz}, 1 \mathrm{H}), 4.75(\mathrm{~m}, \mathrm{br}, 1 \mathrm{H}), 3.44(\mathrm{dd}, J=12.7$ and $4.9 \mathrm{~Hz}, 1 \mathrm{H})$, $2.96(\mathrm{dd}, J=12.7$ and $8.8 \mathrm{~Hz}, 1 \mathrm{H}), 2.43(\mathrm{dd}, J=12.7$ and $8.5 \mathrm{~Hz}$, $1 \mathrm{H}), 2.35(\mathrm{dd}, J=12.7$ and $7.6 \mathrm{~Hz}, 1 \mathrm{H}), 2.26(\mathrm{~s}, 3 \mathrm{H}), 1.55(\mathrm{~s}, 9 \mathrm{H})$. ${ }^{13} \mathrm{C}$ NMR $\left(100 \mathrm{MHz}, \mathrm{CDCl}_{3}\right) \delta 175.4,158.0$ (br), 142.4, 139.6, 139.4, 137.0, 135.3, 132.9, 131.7, 129.9 (2C), 129.1, 128.8 (2C), 128.7, 127.8, 127.3 (4C), 127.3, 127.2, 127.0 (2C), 123.6, 109.4, 81.0, 68.7, 61.2 , 44.8, 44.2, 41.0, 28.4 (3C), 21.0. HRMS (ESI) $m / z$ : $582.2720[\mathrm{M}+\mathrm{Na}]^{+}$; calcd for $\mathrm{C}_{36} \mathrm{H}_{37} \mathrm{~N}_{3} \mathrm{NaO}_{3}{ }^{+}, 582.2727$.

$\left(3 S^{*}\right)$-tert-Butyl $5^{\prime}$-([1,1'-biphenyl]-4-ylmethyl)-1-benzyl-5-bro mo-2-oxospiro[indoline-3, $3^{\prime}$-pyrazolidine]-1' ${ }^{\prime}$-carboxylate (61 and $\left.\mathbf{6} \mathbf{l}^{\prime}\right)$. Prepared according to TP-C starting from compound 5e and $p$-Br-biphenyl, and purified by FC (hexane : ethyl acetate $7: 3$ ). Yellow foam (34\% yield, unseparable diastereoisomers, $1: 1 \mathrm{dr}$ ). ${ }^{1} \mathrm{H}$ NMR (400 $\mathrm{MHz}, \mathrm{CDCl}_{3}, 7: 3$ mixture of diastereoisomers) $\delta 7.67-7.20(\mathrm{~m}, 16 \mathrm{H}), 7.13(\mathrm{~d}, J=2.1 \mathrm{~Hz}, 0.7 \mathrm{H}), 6.85(\mathrm{~m}, \mathrm{br}$, $0.3 \mathrm{H}), 6.61(\mathrm{~d}, J=8.4 \mathrm{~Hz}, 0.7 \mathrm{H}), 6.50(\mathrm{~d}, J=8.4 \mathrm{~Hz}, 0.3 \mathrm{H}), 5.07-$ $4.93(\mathrm{~m}, \mathrm{br}, 1.3 \mathrm{H}), 4.85-4.64(\mathrm{~m}, \mathrm{br}, 1.7 \mathrm{H}), 3.21(\mathrm{dd}, J=13.2$ and $5.0 \mathrm{~Hz}, 0.7 \mathrm{H}), 3.29(\mathrm{dd}, J=13.1$ and $4.4 \mathrm{~Hz}, 0.3 \mathrm{H}), 3.14(\mathrm{dd}, J=$ 13.1 and $6.7 \mathrm{~Hz}, 0.3 \mathrm{H}), 2.97(\mathrm{dd}, J=13.2$ and $8.8 \mathrm{~Hz}, 0.7 \mathrm{H}), 2.61$ $(\mathrm{dd}, J=13.2$ and $8.2 \mathrm{~Hz}, 0.3 \mathrm{H}), 2.42(\mathrm{dd}, J=12.6$ and $8.2 \mathrm{~Hz}$, $0.7 \mathrm{H}), 2.37(\mathrm{dd}, J=12.6$ and $7.6 \mathrm{~Hz}, 0.7 \mathrm{H}), 2.21(\mathrm{dd}, J=13.2$ and $7.6 \mathrm{~Hz}, 0.3 \mathrm{H}), 1.56(\mathrm{~m}, 9 \mathrm{H}) .{ }^{13} \mathrm{C}$ NMR (100 MHz, $\mathrm{CDCl}_{3}, 7: 3$ mixture of diastereoisomers) $\delta 175.7$ and 173.7 (1C), $159.0(\mathrm{br})$, 146.1 and 142.2 (1C), 140.9 and 140.2 (1C), 139.7 and 138.7 (1C), 137.0 and $136.8(1 \mathrm{C}), 136.0$ and 135.1 (1C), 134.6 and $134.2(1 \mathrm{C})$, 133.7 and 120.7 (1C), 132.7-126.0 (16C), 112.2 and $110.8(1 \mathrm{C})$, 81.5, 68.3 and $68.1(1 \mathrm{C}), 61.1$ and 60.1 (1C), 44.4 and $43.5(1 \mathrm{C})$, 43.9 and 41.3 (1C), 43.5 and 41.0 (1C), 28.4 and 28.1 (3C). HRMS (ESI) $m / z: 646.1676[\mathrm{M}+\mathrm{Na}]^{+}$; calcd for $\mathrm{C}_{35} \mathrm{H}_{34} \mathrm{BrN}_{3} \mathrm{NaO}_{3}{ }^{+}$, 646.1676 .

$\left(3 S^{*}, 5^{\prime} S^{*}\right)$-tert-Butyl $5^{\prime}$-([1,1'-biphenyl $]-4$-ylmethyl)-1-benzyl6-chloro-2-oxospiro[indoline-3, $3^{\prime}$-pyrazolidine]-1'-carboxylate (6m) and $\left(3 S^{*}, 5^{\prime} R^{*}\right)$-tert-butyl $5^{\prime}$-([1,1'-biphenyl]-4-ylmethyl)-1benzyl-6-chloro-2-oxospiro[indoline-3,3'-pyrazolidine]-1'-

carboxylate $\left(6 \mathrm{~m}^{\prime}\right)$. Prepared according to TP-C starting from compound $\mathbf{5 f}$ and $p$-Br-biphenyl, and purified by FC (hexane : ethyl acetate $7: 3)$. Yellow foam (72\% yield, separated diastereoisomers, $1.5: 1 \mathrm{dr}$ ).

Diastereoisomer 6m. ${ }^{1} \mathrm{H}$ NMR $\left(400 \mathrm{MHz}, \mathrm{CDCl}_{3}\right) \delta 7.62(\mathrm{dd}$, $\mathrm{br}, J=7.9$ and $1.5 \mathrm{~Hz}, 4 \mathrm{H}), 7.48(\mathrm{t}, J=7.8 \mathrm{~Hz}, 2 \mathrm{H}), 7.44-7.23(\mathrm{~m}$, $9 \mathrm{H}), 6.90$ (dd, br, $J=7.8$ and $1.2 \mathrm{~Hz}, 1 \mathrm{H}), 6.75-6.65(\mathrm{~m}, \mathrm{br}, 1 \mathrm{H})$, 6.65 (d, br, $J=1.5 \mathrm{~Hz}, 1 \mathrm{H}), 5.02(\mathrm{~m}, \mathrm{br}, 1 \mathrm{H}), 4,91-4.68$ (m, br, $2 \mathrm{H}), 3.21(\mathrm{dd}, J=13.5$ and $4.4 \mathrm{~Hz}, 1 \mathrm{H}), 3.16(\mathrm{dd}, J=13.5$ and $7.0 \mathrm{~Hz}, 1 \mathrm{H}), 2.59(\mathrm{dd}, J=12.9$ and $7.9 \mathrm{~Hz}, 1 \mathrm{H}), 2.20(\mathrm{dd}, J=12.9$ and $7.9 \mathrm{~Hz}, 1 \mathrm{H}), 1.57(\mathrm{~s}, 9 \mathrm{H}) .{ }^{13} \mathrm{C} \mathrm{NMR}\left(100 \mathrm{MHz}, \mathrm{CDCl}_{3}\right) \delta 177.1$, 157.3, 145.6, 141.4, 140.5, 137.4 (2C), 136.4, 135.8, 131.1 (2C), 129.6 (2C), 129.5 (2C), 128.5, 128.0 (3C), 127.8 (2C), 127.7 (2C), 124.3, 123.3, 110.6, 81.6, 66.9, 60.9, 44.2, 41.1 (2C), 29.1 (3C). 
HRMS (ESI) $m / z: 602.2189[\mathrm{M}+\mathrm{Na}]^{+}$; calcd for $\mathrm{C}_{35} \mathrm{H}_{34} \mathrm{ClN}_{3^{-}}$ $\mathrm{NaO}_{3}{ }^{+}$, 602.2181. Diastereoisomer $6 \mathrm{~m}^{\prime} .{ }^{1} \mathrm{H}$ NMR $(300 \mathrm{MHz}$, $\left.\mathrm{CDCl}_{3}\right) \delta 7.54(\mathrm{t}, \mathrm{br}, J=7.9 \mathrm{~Hz}, 4 \mathrm{H}), 7.42(\mathrm{t}, \mathrm{br}, J=7.8 \mathrm{~Hz}, 2 \mathrm{H})$, 7.37-7.20 (m, 9H), $6.95(\mathrm{~d}, \mathrm{br}, J=7.8 \mathrm{~Hz}, 1 \mathrm{H}), 6.91(\mathrm{t}, \mathrm{br}, J=$ $7.8 \mathrm{~Hz}, 1 \mathrm{H}), 6.70(\mathrm{~s}, \mathrm{br}, 1 \mathrm{H}), 4,94(\mathrm{~d}, J=15.6 \mathrm{~Hz}, 1 \mathrm{H}), 4,75(\mathrm{~d}, J=$ $15.6 \mathrm{~Hz}, 1 \mathrm{H}), 4.69$ (m, br, $1 \mathrm{H}), 3.39$ (dd, $J=12.7$ and $4.9 \mathrm{~Hz}, 1 \mathrm{H}$ ), $2.91(\mathrm{dd}, J=12.7$ and $8.8 \mathrm{~Hz}, 1 \mathrm{H}), 2.39(\mathrm{dd}, J=12.7$ and $8.8 \mathrm{~Hz}$, $1 \mathrm{H}), 2.29$ (dd, $J=12.7$ and $7.8 \mathrm{~Hz}, 1 \mathrm{H}), 1.49$ (s, 9H). ${ }^{13} \mathrm{C}$ NMR $\left(100 \mathrm{MHz}, \mathrm{CDCl}_{3}\right) \delta 174.2,156.0,143.2,140.9,139.7,136.8$ (2C), 134.8, 134.6, 129.9 (2C), 129.1 (2C), 128.8 (2C), 128.1, 127.4 (2C), 127.3 (3C), 127.1 (2C), 123.7, 123.2, 110.4, 81.2, 67.8, 61.1, 44.6, 44.4, 40.8, 28.4 (3C). HRMS (ESI) $m / z: 602.2188[\mathrm{M}+\mathrm{Na}]^{+}$; calcd for $\mathrm{C}_{35} \mathrm{H}_{34} \mathrm{ClN}_{3} \mathrm{NaO}_{3}{ }^{+}, 602.2181$.

$\left(3 S^{*}, 5^{\prime} S^{*}\right)$-tert-Butyl $5^{\prime}$-([1,1'-biphenyl]-4-ylmethyl)-1-benzyl-2oxo-7-(trifluoromethyl)spiro[indoline-3,3' -pyrazolidine]-1' -carboxylate (6n) and $\left(3 S^{*}, 5^{\prime} R^{*}\right)$-tert-butyl $5^{\prime}-\left(\left[1,1^{\prime}\right.\right.$-biphenyl $]-4$-ylmethyl)1-benzyl-2-oxo-7-(trifluoromethyl)spiro[indoline-3,3'-pyrazolidine ]-1'-carboxylate $\left(\mathbf{6} \mathbf{n}^{\prime}\right)$. Prepared according to TP-C starting from compound $\mathbf{5 g}$ and $p$-Br-biphenyl, and purified by FC (hexane : ethyl acetate $7: 3$ ). Yellow foam (47\% yield, separated diastereoisomers, $1.4: 1 \mathrm{dr}$ ).

Diastereoisomer 6n. ${ }^{1} \mathrm{H}$ NMR $\left(300 \mathrm{MHz}, \mathrm{CDCl}_{3}\right) \delta 7.66-7.54$ (m, 4H), 7.54-7.33 (m, 6H), 7.31-6.93 (m, 7H), $6.83(\mathrm{~m}, \mathrm{br}, 1 \mathrm{H})$, 5.15 (d, br, $J=16.6 \mathrm{~Hz}, 1 \mathrm{H}), 5.10-4.94$ (m, 2H), 3.24-3.08 (m, $2 \mathrm{H}), 2.59(\mathrm{dd}, J=12.7$ and $7.8 \mathrm{~Hz}, 1 \mathrm{H}), 2.20(\mathrm{dd}, J=12.7$ and $7.8 \mathrm{~Hz}, 1 \mathrm{H}), 1.47$ (s, 9H). ${ }^{13} \mathrm{C}$ NMR (100 MHz, $\left.\mathrm{CDCl}_{3}\right) \delta 177.7$, 156.9 (br), 142.0, 140.8, 136.7, 136.2, 132.6, 130.6 (2C), 128.9 (2C), 128.4 (2C), 128.0 (q, $J=5.9 \mathrm{~Hz}), 127.4$ (4C), 127.0 (2C), $126.9,126.2,125.7(2 \mathrm{C}), 123.0(\mathrm{q}, J=272.8 \mathrm{~Hz}), 122.3,113.2(\mathrm{q}, J$ $=33.9 \mathrm{~Hz}$ ), 81.0, 64.7, 60.1, 45.7, 45.0, 40.2, 28.3 (3C). HRMS (ESI) $m / z: 636.2448[\mathrm{M}+\mathrm{Na}]^{+}$; calcd for $\mathrm{C}_{36} \mathrm{H}_{34} \mathrm{~F}_{3} \mathrm{~N}_{3} \mathrm{NaO}_{3}{ }^{+}$, 636.2444. Diastereoisomer 6n'. ${ }^{1} \mathrm{H} \mathrm{NMR}\left(300 \mathrm{MHz}, \mathrm{CDCl}_{3}\right.$ ) $\delta 7.60-7.52(\mathrm{~m}, 5 \mathrm{H}), 7.43(\mathrm{t}, \mathrm{br}, J=7.8 \mathrm{~Hz}, 2 \mathrm{H}), 7.37-7.02(\mathrm{~m}$, $11 \mathrm{H}), 5.22(\mathrm{~d}, J=16.6 \mathrm{~Hz}, 1 \mathrm{H}), 5.17(\mathrm{~d}, J=16.6 \mathrm{~Hz}, 1 \mathrm{H}), 4.77(\mathrm{~m}$, br, $1 \mathrm{H}), 3.40(\mathrm{dd}, J=12.7$ and $4.9 \mathrm{~Hz}, 1 \mathrm{H}), 2.94(\mathrm{dd}, J=12.7$ and $8.8 \mathrm{~Hz}, 1 \mathrm{H}), 2.45(\mathrm{dd}, J=12.7$ and $7.8 \mathrm{~Hz}, 1 \mathrm{H}), 2.37(\mathrm{dd}, J=12.7$ and $7.8 \mathrm{~Hz}, 1 \mathrm{H}), 1.52(\mathrm{~s}, 9 \mathrm{H}) .{ }^{13} \mathrm{C} \mathrm{NMR}\left(100 \mathrm{MHz}, \mathrm{CDCl}_{3}\right) \delta 175.9$, 150.5 (br), 140.9, 140.1, 139.7, 136.7, 135.6, 134.8, 129.9 (2C), 128.8 (2C), 128.5 (2C), 127.4-127.0 (7C), 126.5, 125.6 (2C), 123.2 $(\mathrm{q}, J=272.8 \mathrm{~Hz}), 123.1,113.6(\mathrm{q}, J=33.9 \mathrm{~Hz}), 81.3,66.3,61.2$, 46.0, 44.9, 40.7, 28.4 (3C). HRMS (ESI) $m / z: 636.2449[\mathrm{M}+\mathrm{Na}]^{+}$; calcd for $\mathrm{C}_{36} \mathrm{H}_{34} \mathrm{~F}_{3} \mathrm{~N}_{3} \mathrm{NaO}_{3}{ }^{+}, 636.2444$.

\section{Analytical data of other new compounds}

(E)-3-(3-([1,1'-Biphenyl]-4-yl)allyl)-1-methylindolin-2-one (3b). ${ }^{1} \mathrm{H}$ NMR $\left(300 \mathrm{MHz}, \mathrm{CDCl}_{3}\right) \delta 7.64-7.15(\mathrm{~m}, 11 \mathrm{H}), 7.03(\mathrm{t}, J=$ $7.8 \mathrm{~Hz}, 1 \mathrm{H}), 6.82(\mathrm{~d}, \mathrm{br}, J=7.8 \mathrm{~Hz}, 1 \mathrm{H}), 6.49(\mathrm{~d}, J=15.6 \mathrm{~Hz}, 1 \mathrm{H})$, 6.22 (ddd, $J=15.6,7.8$ and $5.8 \mathrm{~Hz}, 1 \mathrm{H}$ ), 3.57 (dd, $J=7.8$ and $4.9 \mathrm{~Hz}, 1 \mathrm{H}), 3.14(\mathrm{~s}, 3 \mathrm{H}), 3.03(\mathrm{dt}, \mathrm{br}, J=14.7$ and $5.4 \mathrm{~Hz}, 1 \mathrm{H})$, $2.68(\mathrm{dt}, J=14.7$ and $7.8 \mathrm{~Hz}, 1 \mathrm{H}) .{ }^{13} \mathrm{C} \mathrm{NMR}\left(75 \mathrm{MHz}, \mathrm{CDCl}_{3}\right.$ ) $\delta 177.2,144.3,140.7,140.1,137.8,136.2,128.8$ (4C), 128.1, 127.2 (4C), 127.0 (2C), 126.1, 124.3, 122.4, 108.1, 45.6, 34.4, 26.2. HRMS (ESI) $m / z: 362.1519[\mathrm{M}+\mathrm{Na}]^{+}$; calcd for $\mathrm{C}_{24} \mathrm{H}_{21} \mathrm{NNaO}^{+}$, 362.1515 .

(E)-tert-Butyl 2-(3-(3-([1,1'-biphenyl]-4-yl)allyl)-1-benzyl-2-oxo indolin-3-yl)hydrazinecarboxylate (7). ${ }^{1} \mathrm{H}$ NMR $(400 \mathrm{MHz}$,
$\left.\mathrm{CDCl}_{3}\right) \delta 7.61-7.21(\mathrm{~m}, 15 \mathrm{H}), 7.18(\mathrm{t}, \mathrm{br}, J=7.2 \mathrm{~Hz}, 1 \mathrm{H}), 7.05(\mathrm{t}$, br, $J=7.5 \mathrm{~Hz}, 1 \mathrm{H}), 6.66(\mathrm{~d}, J=7.6 \mathrm{~Hz}, 1 \mathrm{H}), 6.46(\mathrm{~d}, \mathrm{br}, J=16.0$, 1H), 6.04 (m, br, 1H), 5.96 (m, br, 1H), 5.90 (ddd, $J=16.0,8.9$ and $6.5 \mathrm{~Hz}, 1 \mathrm{H}), 5.10(\mathrm{~d}, \mathrm{br}, J=15.7 \mathrm{~Hz}, 1 \mathrm{H}), 4.70(\mathrm{~d}, \mathrm{br}, J=$ $15.7 \mathrm{~Hz}, 1 \mathrm{H}), 2.94(\mathrm{dd}, J=12.6$ and $5.8 \mathrm{~Hz}, 1 \mathrm{H}), 2.86(\mathrm{dd}, J=12.6$ and $8.5 \mathrm{~Hz}, 1 \mathrm{H}), 1.36(\mathrm{~s}, 9 \mathrm{H}) .{ }^{13} \mathrm{C} \mathrm{NMR}\left(100 \mathrm{MHz}, \mathrm{CDCl}_{3}\right) \delta 177.4$, 156.0, 144.2, 143.1, 141.9, 137.9, 136.8, 134.5, 129.2, 128.8 (2C), 128.7 (2C), 127.6-126.8 (9C), 125.2, 125.1, 122.8, 122.1, 109.3, 83.8, 68.5, 43.9, 38.9, 28.2 (3C). HRMS (ESI) $m / z: 568.2177[\mathrm{M}+$ $\mathrm{Na}]^{+}$; calcd for $\mathrm{C}_{35} \mathrm{H}_{35} \mathrm{~N}_{3} \mathrm{NaO}_{3}{ }^{+}, 568.2571$.

(E)-tert-Butyl 2-(1-benzyl-2-oxo-3-(prop-1-en-1-yl)indolin-3-yl) hydrazinecarboxylate (8). ${ }^{1} \mathrm{H}$ NMR $\left(400 \mathrm{MHz} \mathrm{CDCl}_{3}\right) \delta 7.45(\mathrm{~d}, J$ $=7.8 \mathrm{~Hz}, 1 \mathrm{H}), 7.35-7.19(\mathrm{~m}, 5 \mathrm{H}), 7.15(\mathrm{t}, J=7.8 \mathrm{~Hz}, 1 \mathrm{H}), 7.03(\mathrm{t}, J$ $=7.8 \mathrm{~Hz}, 1 \mathrm{H})), 6.65(\mathrm{~d}, J=7.8 \mathrm{~Hz}, 1 \mathrm{H}), 6.16-5.87(\mathrm{~m}, \mathrm{br}, 2 \mathrm{H})$, $5.78(\mathrm{dq}, J=15.6$ and $5.9 \mathrm{~Hz}, 1 \mathrm{H}), 5.71(\mathrm{~d}, J=15.6 \mathrm{~Hz}, 1 \mathrm{H}), 4.91$ $(\mathrm{m}, 2 \mathrm{H}), 1.69(\mathrm{~d}, J=5.9 \mathrm{~Hz}, 3 \mathrm{H}), 1.31(\mathrm{~s}, 9 \mathrm{H}) .{ }^{13} \mathrm{C} \mathrm{NMR}(75 \mathrm{MHz}$, $\left.\mathrm{CDCl}_{3}\right) \delta 177.4,156.1,143.2,142.8,135.7,130.9,129.1,128.8$ (2C), 127.6, 127.2 (2C), 127.0, 125.7, 122.7, 109.2, 80.5, 70.0, 43.8, 28.1 (3C), 18.1. HRMS (ESI) $m / z: 416.1941[\mathrm{M}+\mathrm{Na}]^{+}$; calcd for $\mathrm{C}_{23} \mathrm{H}_{27} \mathrm{~N}_{3} \mathrm{NaO}_{3}{ }^{+}, 416.1945$.

3-Allyl-1-benzylindolin-2-one (9). ${ }^{1} \mathrm{H}$ NMR (300 MHz, $\left.\mathrm{CDCl}_{3}\right)$ $\delta 7.34-7.21(\mathrm{~m}, 6 \mathrm{H}), 7.15(\mathrm{t}, \mathrm{br}, J=7.8 \mathrm{~Hz}, 1 \mathrm{H}), 7.00(\mathrm{t}, \mathrm{br}, J=$ $7.8 \mathrm{~Hz}, 1 \mathrm{H}), 6.70(\mathrm{~d}, J=7.8 \mathrm{~Hz}, 1 \mathrm{H}), 5.75(\mathrm{~m}, 1 \mathrm{H}), 5.13(\mathrm{~d}, \mathrm{br}, J=$ $15.6 \mathrm{~Hz}, 1 \mathrm{H}), 5.05$ (d, br, $J=10.7 \mathrm{~Hz}, 1 \mathrm{H}), 4.99$ (d, $J=15.6 \mathrm{~Hz}$, $1 \mathrm{H}), 4.82$ (d, $J=15.6 \mathrm{~Hz}, 1 \mathrm{H}), 3.60(\mathrm{~m}, 1 \mathrm{H}), 2.88(\mathrm{~m}, 1 \mathrm{H}), 2.65$ $(\mathrm{dt}, J=14.6$ and $7.8 \mathrm{~Hz}, 1 \mathrm{H}) .{ }^{13} \mathrm{C} \mathrm{NMR}\left(75 \mathrm{MHz}, \mathrm{CDCl}_{3}\right) \delta 177.3$, 143.5, 135.9, 133.9, 128.8 (2C), 128.6, 127.9, 127.6 (2C), 127.4, 124.2, 122.3, 118.2, 109.0, 45.2, 43.7, 35.0. HRMS (ESI) $m / z$ : $286.1200[\mathrm{M}+\mathrm{Na}]^{+}$; calcd for $\mathrm{C}_{18} \mathrm{H}_{17} \mathrm{NNaO}^{+}, 286.1202$.

Di-tert-butyl 1-(3-allyl-1-benzyl-2-oxoindolin-3-yl)hydrazine1,2-dicarboxylate (10). ${ }^{1} \mathrm{H}$ NMR $\left(300 \mathrm{MHz}, \mathrm{CDCl}_{3}\right) \delta$ 7.40-7.19 $(\mathrm{m}, 6 \mathrm{H}), 7.13$ (t, br, $J=7.8 \mathrm{~Hz}, 1 \mathrm{H}), 6.97(\mathrm{t}, \mathrm{br}, J=7.8 \mathrm{~Hz}, 1 \mathrm{H})$, 6.59 (d, br, $J=7.8 \mathrm{~Hz}, 1 \mathrm{H}), 5.46(\mathrm{~m}, 1 \mathrm{H}), 5.34(\mathrm{~m}, \mathrm{br}, 1 \mathrm{H}), 5.02$ (d, br, $J=16.6 \mathrm{~Hz}, 1 \mathrm{H}), 4.93$ (d, br, $J=10.0 \mathrm{~Hz}, 1 \mathrm{H}), 4.88(\mathrm{~d}, \mathrm{br}, J$ $=15.6 \mathrm{~Hz}, 1 \mathrm{H}), 4.79(\mathrm{~d}, \mathrm{br}, J=15.6 \mathrm{~Hz}, 1 \mathrm{H}), 2.86(\mathrm{dd}, \mathrm{br}, J=13.7$ and $6.8 \mathrm{~Hz}, 1 \mathrm{H}), 2.75(\mathrm{dd}, \mathrm{br}, J=13.7$ and $7.8 \mathrm{~Hz}, 1 \mathrm{H}), 1.39(\mathrm{~s}$, 18H). ${ }^{13} \mathrm{C} \mathrm{NMR} \mathrm{(75} \mathrm{MHz,} \mathrm{CDCl}_{3}$ ) $\delta$ 176.7, 153.3 (2C), 143.5, 135.8, 131.4, 129.2, 128.6 (2C), 127.4 (3C), 126.7, 125.5, 122.2, 119.8, 109.1, 83.3 (2C), 67.5, 43.8, 40.8, 27.9 (6C). HRMS (ESI) m/ $z: 516.2473[\mathrm{M}+\mathrm{Na}]^{+}$; calcd for $\mathrm{C}_{28} \mathrm{H}_{35} \mathrm{~N}_{3} \mathrm{NaO}_{5}{ }^{+}, 516.2469$.

(E)-Di-tert-butyl 1-(3-(3-([1,1'-biphenyl]-4-yl)allyl)-1-benzyl-2oxoindolin-3-yl)hydrazine-1,2-dicarboxylate (11). ${ }^{1} \mathrm{H}$ NMR (400 $\left.\mathrm{MHz} \mathrm{CDCl}_{3}\right) \delta 7.59(\mathrm{~d}, \mathrm{br}, J=8.4 \mathrm{~Hz}, 2 \mathrm{H}), 7.51-6.96(\mathrm{~m}, 16 \mathrm{H})$, $6.59(\mathrm{~d}, J=7.8 \mathrm{~Hz}, 1 \mathrm{H}), 6.44(\mathrm{~d}, J=15.8 \mathrm{~Hz}, 1 \mathrm{H}), 5.87$ (ddd, $J=$ $15.8,8.5$ and $6.4 \mathrm{~Hz}, 1 \mathrm{H}), 5.10(\mathrm{~d}, J=16.1 \mathrm{~Hz}, 1 \mathrm{H}), 4.63(\mathrm{~d}, J=$ $16.1 \mathrm{~Hz}, 1 \mathrm{H}), 3.10(\mathrm{dd}, J=13.2$ and $6.4 \mathrm{~Hz}, 1 \mathrm{H}), 3.01(\mathrm{dd}, J=13.2$ and $8.5 \mathrm{~Hz}, 1 \mathrm{H}), 1.46(\mathrm{~s}, 18 \mathrm{H}) .{ }^{13} \mathrm{C} \mathrm{NMR}\left(100 \mathrm{MHz}, \mathrm{CDCl}_{3}\right)$ $\delta 177.3,154.0$ (2C), 144.2, 141.5, 140.7, 136.9, 136.2, 134.9, 130.1, 129.4 (2C), 129.2 (2C), 127.9 (2C), 127.8 (4C), 127.6 (2C), 127.4 (2C), 126.1, 124.5, 123.7, 123.0, 109.9, 84.1 (2C), 68.5, 44.5, 40.9, 28.6 (6C). HRMS (ESI) $\mathrm{m} / z: 668.3097[\mathrm{M}+\mathrm{Na}]^{+}$; calcd for $\mathrm{C}_{40} \mathrm{H}_{43} \mathrm{~N}_{3} \mathrm{NaO}_{5}{ }^{+}, 668.3095$.

tert-Butyl 2-(1-benzyl-3-(2-methylbut-3-en-2-yl)-2-oxoindolin3-yl)hydrazinecarboxylate (12). ${ }^{1} \mathrm{H}$ NMR (400 $\left.\mathrm{MHz}, \mathrm{CDCl}_{3}\right)$ $\delta 7.50(\mathrm{~d}, J=7.7 \mathrm{~Hz}, 1 \mathrm{H}), 7.37-7.24(\mathrm{~m}, 5 \mathrm{H}), 7.18(\mathrm{t}, J=7.8 \mathrm{~Hz}$, $1 \mathrm{H}), 7.01(\mathrm{t}, J=7.8 \mathrm{~Hz}, 1 \mathrm{H}), 6.66(\mathrm{~d}, J=7.7 \mathrm{~Hz}, 1 \mathrm{H}), 6.26(\mathrm{dd}, J=$ 17.5 and $10.8 \mathrm{~Hz}, 1 \mathrm{H}), 5.60$ (m, br, $1 \mathrm{H}), 5.19$ (d, $J=10.8 \mathrm{~Hz}, 1 \mathrm{H})$, 
$5.14(\mathrm{~d}, \mathrm{br}, J=17.5 \mathrm{~Hz}, 1 \mathrm{H}), 5.02-4.78(\mathrm{~m}, 3 \mathrm{H}), 1.26$ (s, br, 9H), $1.24(\mathrm{~s}, 3 \mathrm{H}), 1.08(\mathrm{~s}, 3 \mathrm{H}) .{ }^{13} \mathrm{C} \mathrm{NMR}\left(100 \mathrm{MHz}, \mathrm{CDCl}_{3}\right) \delta 178.5$, 156.4, 144.9, 142.7, 136.5, 129.6, 129.4 (2C), 128.2, 128.1, 127.9 (2C), 127.3, 122.4, 115.7, 109.3, 80.9, 74.9, 44.6, 43.0, 28.7 (3C), 23.7, 21.4. HRMS (ESI) $m / z: 444.2261[\mathrm{M}+\mathrm{Na}]^{+}$; calcd for $\mathrm{C}_{25} \mathrm{H}_{31} \mathrm{~N}_{3} \mathrm{NaO}_{3}{ }^{+}, 444.2258$.

$\left(3 S^{*}, 5^{\prime} R^{*}\right)-$ and $\left(3 S^{*}, 5^{\prime} S^{*}\right)$-tert-Butyl $5^{\prime}-\left(\left[1,1^{\prime}\right.\right.$-biphenyl $]-4-$ ylmethyl)-1-benzyl-4' $\mathbf{4}^{\prime} \mathbf{4}^{\prime}$-dimethyl-2-oxospiro[indoline-3,3' ${ }^{\prime}$-pyrazolidine]-1'-carboxylate (13). ${ }^{1} \mathrm{H} \mathrm{NMR}\left(300 \mathrm{MHz}, \mathrm{CDCl}_{3}\right.$, cis : trans $7: 3$ diastereoisomeric mixture) $\delta 7.64-7.11(\mathrm{~m}, 16 \mathrm{H}), 7.01$ $(\mathrm{t}, \mathrm{br}, J=7.8 \mathrm{~Hz}, 0.3 \mathrm{H}), 6.98(\mathrm{t}, \mathrm{br}, J=7.8 \mathrm{~Hz}, 0.7 \mathrm{H}), 6.65(\mathrm{~d}, J=$ $7.8 \mathrm{~Hz}, 0.7 \mathrm{H}), 6.61(\mathrm{~d}, J=7.8 \mathrm{~Hz}, 0.3 \mathrm{H}), 5.27-5.02(\mathrm{~m}, 2 \mathrm{H}), 4.81$ $(\mathrm{m}, 0.3 \mathrm{H}), 4.77(\mathrm{dd}, J=10.7$ and $3.9 \mathrm{~Hz}, 0.7 \mathrm{H}), 4.66(\mathrm{~d}, \mathrm{br}, J=$ $15.6 \mathrm{~Hz}, 0.3 \mathrm{H}$ ), 4.46 (d, br, $J=15.6 \mathrm{~Hz}, 0.7 \mathrm{H}), 3.69$ (dd, $J=13.7$ and $3.9 \mathrm{~Hz}, 0.7 \mathrm{H}), 2.86(\mathrm{dd}, J=13.7$ and $10.7 \mathrm{~Hz}, 0.7 \mathrm{H}), 2.74-$ $2.56(\mathrm{~m}, 0.6 \mathrm{H}), 1.57(\mathrm{~s}, 6.3 \mathrm{H}), 1.55(\mathrm{~s}, 0.9 \mathrm{H}), 1.50(\mathrm{~s}, 0.9 \mathrm{H}), 1.38$ $(\mathrm{s}, 2.1 \mathrm{H}), 1.31(\mathrm{~s}, 2.7 \mathrm{H}), 1.25(\mathrm{~s}, 2.1 \mathrm{H}) .{ }^{13} \mathrm{C} \mathrm{NMR}\left(75 \mathrm{MHz}, \mathrm{CDCl}_{3}\right)$ $\delta 176.5$ and 169.6 (1C), 157.8 and 156.0 (1C), 144.6 and 143.3 (1C), 140.8, 139.1, 137.7 and 136.7 (1C), 135.8 and 123.1 (2C), 129.8-125.2 $(x \mathrm{C}), 122.6$ and $121.9(1 \mathrm{C}), 109.3$ and $109.0(1 \mathrm{C})$, 81.1 and 80.4 (1C), 73.2 and $69.4(1 \mathrm{C}), 67.0,49.3,43.7$ and 43.6 (1C), 36.4 and 34.1 (1C), 28.5 and 28.1 (3C), 25.9 and 20.4 (1C), 22.2 and 18.0 (1C). HRMS (ESI) $\mathrm{m} / z$ : $596.2888[\mathrm{M}+\mathrm{Na}]^{+}$; calcd for $\mathrm{C}_{37} \mathrm{H}_{39} \mathrm{~N}_{3} \mathrm{NaO}_{3}{ }^{+}$, 596.2884.

\section{Conflicts of interest}

The authors declare no competing financial interest.

\section{References}

1 L. M. Blair and J. Sperry, Natural Products Containing a Nitrogen-Nitrogen Bond, J. Nat. Prod., 2013, 76, 794-812.

2 M. S. Ali, F. Ahmad, V. U. Ahmad, I. Azhar and K. Usmanghani, Unusual Chemical Constituents of Lotus garcinia (Fabaceae), Turk. J. Chem., 2001, 25, 107-112.

3 D. Secci, A. Bolasco, P. Chimenti and S. Carradori, The State of the Art of Pyrazole Derivatives as Monoamine Oxidase Inhibitors and Antidepressant/Anticonvulsant Agents, Curr. Med. Chem., 2011, 18, 5114-5144.

4 B. Parashar, A. Jain, S. Bharadwaj and V. K. Sharma, Synthesis and pharmacological properties of some novel pyrazolidine and pyrazole derivatives, Med. Chem. Res., 2012, 21, 1692-1699.

5 E. Frank, Z. Mucsi, I. Zupko, B. Rethy, G. Falkay, G. Schneider and J. Wolfling, Efficient Approach to Androstene-Fused Arylpyrazolines as Potent Antiproliferative Agents. Experimental and Theoretical Studies of Substituent Effects on BF3-Catalyzed Intramolecular [3+2] Cycloadditions of Olefinic Phenylhydrazones, J. Am. Chem. Soc., 2009, 131, 3894-3904.

6 M. J. Kornet and R. J. Garrett, Synthesis of 1-phenyl-2(phenylcarbamoyl)pyrazolidines as potential anticonvulsant agents, J. Pharm. Sci., 1979, 68, 377-378.

7 (a) J. H. Ahn, J. A. Kim, H. M. Kim, H. M. Kwon, S. C. Huh, S. D. Rhee, K. R. Kim, S. D. Yang, S. D. Park, J. M. Lee, S. S. Kim and H. G. Cheon, Synthesis and evaluation of pyrazolidine derivatives as dipeptidyl peptidase IV (DP-IV) inhibitors, Bioorg. Med. Chem. Lett., 2005, 15, 1337-1340; (b) H. G. Cheon, S. S. Kim, K. R. Kim, S. D. Rhee, S. D. Yang, J. H. Ahn, S. D. Park, J. M. Lee, W. H. Jung, H. S. Lee and H. Y. Kim, Inhibition of dipeptidyl peptidase IV by novel inhibitors with pyrazolidine scaffold, Biochem. Pharmacol., 2005, 70, 22-29.

8 G. X. Yang, L. L. Chang, Q. Truong, G. A. Doherty, P. A. Magriotis, S. E. de Laszlo, B. Li, M. MacCoss, U. Kidambi, L. A. Egger, E. McCauley, G. V. Riper, R. A. Mumford, J. A. Schmidt and W. K. Hagmann, NTetrahydrofuroyl-(L)-phenylalanine derivatives as potent VLA-4 antagonists, Bioorg. Med. Chem. Lett., 2002, 12, 14971500 .

9 S. H. Ahmed, H. Ahmed, S. H. Ali, S. Fatima, S. Hyder and A. Pasha, Synthesis and anti-bacterial activity of some novel pyrazolidinedione substituted derivatives of 2quinolones, Indo Am. J. Pharm. Res., 2017, 7, 801-823.

10 K. A. Koo, N. D. Kim, Y. S. Chon, M. S. Jung, B. J. Lee, J. H. Kim and W. J. Song, QSAR analysis of pyrazolidine3,5-diones derivatives as Dyrk1A inhibitors, Bioorg. Med. Chem. Lett., 2009, 19, 2324-2328.

11 K. Singh, R. S. N. Munuganti, E. Leblanc, Y. L. Lin, E. Leung, N. Lallous, M. Butler, A. Cherkasov and P. S. Rennie, In silico discovery and validation of potent small-molecule inhibitors targeting the activation function 2 site of human oestrogen receptor $\alpha$, Breast Cancer Res., 2015, 17, 1-17.

12 J. Velcicky, U. Bodendorf, P. Rigollier, R. Epple, D. R. Beisner, D. Guerini, et al., Discovery of the First Potent, Selective, and Orally Bioavailable Signal Peptide Peptidase-Like 2a (SPPL2a) Inhibitor Displaying Pronounced Immunomodulatory Effects In Vivo, J. Med. Chem., 2018, 61, 865-880.

13 Y. Yamashita and S. Kobayashi, Zirconium-Catalyzed Enantioselective [3+2] Cycloaddition of Hydrazones to Olefins Leading to Optically Active Pyrazolidine, Pyrazoline, and 1,3Diamine Derivatives, J. Am. Chem. Soc., 2004, 126, 1127911282.

14 (a) F. Požgan, H. AlMamari, U. Grošelj, J. Svete and B. Štefane, Synthesis of Non-Racemic Pyrazolines and Pyrazolidines by $[3+2]$ Cycloadditions of Azomethine Imines, Molecules, 2018, 23, 3; (b) U. Grošelj, J. Svete, H. Al Mamari, F. Požgan and B. Štefane, Metal-catalyzed [3+2] cycloadditions of azomethine imines, Chem. Heterocycl. Compd., 2018, 54, 214-240.

15 (a) B. R. Rosen, J. E. Ney and J. P. Wolfe, Use of Aryl Chlorides as Electrophiles in Pd-Catalyzed Alkene Difunctionalization Reactions, J. Org. Chem., 2010, 75, 2756-2759; (b) J. P. Wolfe and N. C. Giampietro, Stereoselective Synthesis of cis- or trans-3,5-Disubstituted Pyrazolidines via PdCatalyzed Carboamination Reactions: Use of Allylic Strain to Control Product Stereochemistry Through N-Substituent Manipulation, J. Am. Chem. Soc., 2008, 130, 12907-12911.

16 L. O. Davis, Recent Developments in the Synthesis and Applications of Pyrazolidines. A Review, Org. Prep. Proced. Int. , 2013, 45, 437-464.

17 (a) G. Rainoldi, F. Begnini, M. de Munnik, L. Lo Presti, C. M. L. Vande Velde, R. Orru, G. Lesma, E. Ruijter and 
A. Silvani, Sequential Multicomponent Strategy for the Diastereoselective Synthesis of Densely Functionalized Spirooxindole-Fused Thiazolidines, ACS Comb. Sci., 2018, 20, 98-105; (b) G. Rainoldi, M. Faltracco, C. Spatti, A. Silvani and G. Lesma, Organocatalytic Access to Enantioenriched Spirooxindole-Based 4-Methyleneazetidines, Molecules, 2017, 22, 2016; (c) G. Rainoldi, M. Faltracco, L. Lo Presti, A. Silvani and G. Lesma, Highly diastereoselective entry into chiral spirooxindole-based 4-methyleneazetidines via formal [2+2] annulation reaction, Chem. Commun., 2016, 52, 11575-11578; (d) G. Rainoldi, F. Begnini, A. Silvani and G. Lesma, Efficient Synthesis of Spirooxindole-fused 3-Thiazoline Derivatives by a One-Pot Asinger-Type Reaction, Synlett, 2016, 27, 28312835; (e) G. Rainoldi, A. Sacchetti, A. Silvani and G. Lesma, Organocatalytic vinylogous Mannich reaction of trimethylsiloxyfuran with isatin-derived benzhydryl-ketimines, Org. Biomol. Chem., 2016, 14, 7768-7776; (f) G. Lesma, F. Meneghetti, A. Sadcchetti, M. Stucchi and A. Silvani, Asymmetric Ugi 3CR on isatin-derived ketimine: synthesis of chiral 3,3-disubstituted 3-aminooxindole derivatives, Beilstein J. Org. Chem., 2014, 10, 1383-1389; (g) G. Lesma, N. Landoni, T. Pilati, A. Sacchetti and A. Silvani, Grignard Addition to Imines Derived from Isatine: A Method for the Asymmetric Synthesis of Quaternary 3-Aminooxindoles, J. Org. Chem., 2009, 74, 4537-4541.

18 Design of Hybrid Molecules for Drug Development. ed. M. Decker, Elsevier Ed, 2017.

19 (a) G.-J. Mei and F. Shi, Catalytic asymmetric synthesis of spirooxindoles: recent developments, Chem. Commun., 2018, 54, 6607-6621; (b) G. Meller, T. Berkenbosch, J. C. J. Benningshof, D. Stumpfe and J. Bajorath, Charting Biologically Relevant Spirocyclic Compound Space, Chem.Eur. J., 2017, 23, 703-71; (c) V. Pace, L. Castoldi, A. D. Mamuye, T. Langer and W. Holzera, Chemoselective
Addition of Halomethyllithiums to Functionalized Isatins: A Straightforward Access to Spiro-Epoxyoxindoles, Adv. Synth. Catal., 2016, 358, 172-177.

20 B. Wagner, W. Hiller, H. Ohno and N. Krause, Gold-catalyzed three-component spirocyclization: a one-pot approach to functionalized pyrazolidines, Org. Biomol. Chem., 2016, 14, 1579-1583.

21 (a) N. A. Aslam, S. A. Babu, S. Rani, S. Mahajan, J. Solanki, M. Yasuda and A. Baba, Diastereoselective Construction of 3-Aminooxindoles with Adjacent Stereocenters: Stereocontrolled Addition of $\gamma$-Substituted Allylindiums to Isatin Ketimines, Eur. J. Org. Chem., 2015, 4168-4189; (b) B. Alcaide, P. Almendros and C. Aragoncillo, Indium-Promoted Allylation Reaction of IminoIsatins in Aqueous Media: Synthesis of Quaternary 3Aminooxindoles, Eur. J. Org. Chem., 2010, 2845-2848.

22 P. D. Chaudhari, B. C. Hong and G. H. Lee, Organocatalytic Enantioselective Michael-Michael-Michael-Aldol Condensation Reactions: Control of Six Stereocenters in a QuadrupleCascade Asymmetric Synthesis of Polysubstituted Spirocyclic Oxindoles, Org. Lett., 2017, 19, 6112-6115.

23 D. Ganggang, H. Danfeng, W. Ke-Hu, C. Xiaowei, X. Yanli, M. Junyan, S. Yingpeng, F. Ying and H. Yulai, One-pot preparation of trifluoromethylated homoallylic N-acylhydrazines or $\alpha$-methylene- $\gamma$-lactams from acylhydrazines, trifluoroacetaldehyde methyl hemiacetal, allyl bromide and tin, Org. Biomol. Chem., 2016, 14, 1492-1500.

24 J. E. Thomson, A. F. Kyle, K. B. Ling, S. R. Smith, A. M. Z. Slawin and A. D. Smith, Applications of NHCmediated $\mathrm{O}-$ to C-carboxyl transfer: synthesis of $( \pm)-\mathrm{N}$ benzyl-coerulescine and ( \pm )-horsfiline, Tetrahedron, 2010, 66, 3801-3813.

25 L. Firmansjah and G. C. Fu, Intramolecular Heck Reactions of Unactivated Alkyl Halides, J. Am. Chem. Soc., 2007, 129, 11340-11341. 